\title{
ANA GASCON
}

\section{Géométrie des variétés invariantes d'un difféomorphisme axiome $A$ et transversalité forte}

\author{
Annales de la faculté des sciences de Toulouse $5^{e}$ série, tome 10, \\ $\mathrm{n}^{\mathrm{o}} 2$ (1989), p. 291-324 \\ <http://www.numdam.org/item?id=AFST_1989_5_10_2_291_0>
}

(C) Université Paul Sabatier, 1989, tous droits réservés.

L'accès aux archives de la revue «Annales de la faculté des sciences de Toulouse » (http://picard.ups-tlse.fr/ annales/) implique l'accord avec les conditions générales d'utilisation (http://www.numdam.org/conditions). Toute utilisation commerciale ou impression systématique est constitutive d'une infraction pénale. Toute copie ou impression de ce fichier doit contenir la présente mention de copyright.

\section{NumDam}

Article numérisé dans le cadre du programme Numérisation de documents anciens mathématiques http://www.numdam.org/ 


\title{
Géométrie des Variétés Invariantes d'un Difféomorphisme Axiome A et Transversalité Forte
}

\author{
ANA CASCON ${ }^{(*)(1)(2)(3)}$
}

\begin{abstract}
RÉSUMÉ. - On étudie sur une variété compacte de dimension $n$ la structure de variétés invariantes près des ensembles basiques des diffeomorphismes qui satisfont les conditions "Axiome A" et "Transversalité Forte". On montre qu'il existe un voisinage de toute pièce basique que ne soit pas un puits où les variétés instables varient de façon continue dans la topologie C. On montre aussi que sur les surfaces les puits et les sources sont les seuls points au voisinage desquels la courbure géodesique des courbes invariantes peut ne pas être bornée.
\end{abstract}

\begin{abstract}
We study the structure of the invariant manifolds nearby the basic sets of an axiom $A$ diffeomorphism of a compact $n$-dimensional manifold satisfying the strong transversality condition.

We show that on a surface, the only points in the neighborhood of which the geodesic curvature of the invariant curves may be unbounded are sinks and sources. Examples are given.
\end{abstract}

\section{KEYWORDS (GLOBAL ANALYSIS)}

- Systèmes Dynamiques

- difféomorphisme

- variétés invariantes

- courbure géodésique

(*) L'auteur remercie pour son soutien financier le CNPQ (Brésil).

(1) Université de Dijon, Département de Mathématiques, Campus Universitaire, B.P. 138, 21004 Dijon Cedex.

(2) Université de Tours, Département de Mathématiques, Parc de Grandmont, 37000 Tours

(3) Rua Marques de São Vicente 86/307, 22451 Rio de Janeiro-RJ, Brésil, tél. $(55)(21) 2593242$ 


\section{Introduction et énoncés des théorèmes}

Notre objectif est d'étudier la configuration globale des variétés invariantes associées à un difféomorphisme.

L'existence de variétés invariantes généralise la notion d'orbite d'un champ de vecteurs. On sait qu'un champ de vecteurs définit un feuilletage (singulier). Une question naturelle est donc dans quels cas les variétés invariantes fournissent une lamination sur une variété. Pour cela, on va étudier les points d'accumulation de courbure. D'une façon naïve, on pourrait dire qu'un point d'accumulation de courbure est tel que sur un voisinage les variétés invariantes se "plient" de plus en plus.

Dans les dernières années les difféomorphismes dits de Hénon ont été beaucoup étudiés. Ces difféomorphismes des surfaces contiennent un point homoclinique transverse. Il a été remarqué notamment l'existence d'un ensemble qui paraît être un attracteur assez compliqué, qu'on appelle attracteur étrange.

Au voisinage d'un tel "attracteur" il existe des variétés invariantes qui se "plient beaucoup". On pourrait donc espérer que l'étude des points d'accumulation de courbure pourrait aider à comprendre de tels ensembles.

On dit que $x$ est un point d'accumulation de courbure instable (ou stable) d'une famille $C_{n}$ de variétés invariantes de dimension un s'il existe une suite $x_{n}$ qui converge vers $x$ telle que chaque $x_{n}$ appartienne à une courbe $C_{n}$ et que $K\left(x_{n}\right)$ tend vers l'infini $\left(K\left(x_{n}\right)\right.$ est la courbure géodésique de $C_{n}$ au point $\left.x_{n}\right)$.

On a démontré (voir $[C],[C L]$ ) que tout point d'accumulation de courbure instable des courbes invariantes des difféomorphismes de Morse-Smale sur une surface compacte est un puits.

On verra ici que le même résultat est vrai pour les difféomorphismes sur une surface compacte qui satisfont les conditions "Axiome A" et "Transversalité Forte".

L'étude des sous-variétés de dimension plus grande que un du point de vue de la courbure est plus complexe et ne sera pas envisagé dans ce travail.

Mais l'étude de la structure locale effectuée dans la partie I devrait permettre de généraliser le résultat sur l'accumulation de courbure (démontré dans la partie II) à des variétés de plus grande dimension. 
Géométrie des Variétés Invariantes d'un Difféomorphisme Axiome A et Transversalité

Dans la suite on va d'abord faire quelques rappels et donner quelques définitions. Dans la partie I on étudie la structure locale des variétés invariantes (de dimension quelconque) au voisinage d'une pièce basique.

On démontre une version un peu plus forte d'un théorème de J. Palis $[\mathrm{P}]$.

THÉORÈME 1. - Soit $f$ un difféomorphisme $C^{r}$ qui satisfait les conditions "Axiome $A$ et transversalité forte". Soit $\Lambda$ une pièce basique qui ne soit pas un puits. Alors il existe un voisinage de $\Lambda$ où les variétés instables varient de façon continue dans la topologie $C^{r}$.

Dans la partie II, on démontre le théorème 2 et on donne quelques exemples.

THÉORÈME 2.- Soit $M$ une surface compacte munie d'un difféomorphisme $f$ qui satisfait les conditions "Axiome $A$ et transversalité forte". Tout point d'accumulation de courbure instable est un puits.

Dans la partie III, on regarde quelques exemples dans le bord de l'ensemble qui satisfait l'Axiome A et la condition de Tranversalité Forte. En particulier on donne un exemple de difféomorphisme avec un point hétéroclinique de tangence qui possède une infinité de points d'accumulation de courbure.

Finalement, dans l'appendice, on démontre une version forte du " $\lambda$ lemma".

Je remercie les innombrables suggestions de J. PALIS et R. LANGEvin et l'aide constante de R. Roussarie.

\section{Définitions et rappels}

Soit $f$ un difféomorphisme de classe $C^{r}$ d'une variété compacte $M$ de dimension $n$ sans bord. On choisit une métrique riemannienne sur $M$. Sur l'ensemble $\operatorname{Diff^{r}}(M)$ des difféomorphismes de $M$ de classe $C^{r}$, nous mettons la topologie $C^{r}$.

Dans la partie I, où on étudie la structure locale, on va travailler avec des variétés invariantes de dimension non constante et on veut contrôler la façon dont ces variétés convergent vers une autre variété de dimension plus petite.

Pour donner un sens à ce problème, on va avoir besoin d'une série de définitions.

D'une façon usuelle, on dit qu'une famille de sous-variétés de dimension $k$ varie de façon continue dans la topoligie $C^{r}$ si tout point admet un voisinage 
dans lequel la trace de la famille est une lamination de dimension $k$ et de classe $C^{r}$.

Une lamination $\mathcal{L}$ de classe $C^{r}$ et de dimension $k$ dans une variété $M$ de dimension $n$ est telle que : pour tout $x \in \mathcal{L}$, il existe un voisinage $\mathcal{V}_{x}$ où la lamination est donnée par l'image de $\left(A \times D^{k}\right)$ : le sous ensemble $\mathrm{A}$ de $R^{n-k}$ est compact et $D^{k}$ est le disque compact de $R^{k}$. L'application $\Phi$ : $A \times D^{k} \rightarrow M$ est telle que pour tout $y$ de $A, \Phi_{y}: D^{k} \rightarrow M$ soit un difféomorphisme de classe $C^{r}$ et $y \rightarrow \Phi_{y}$ soit une application continue dans la topologie $C^{r}$. Un plaque de $\mathcal{L}$ est l'image par $\Phi$ de $\{y\} \times D^{k}$. La classe d'équivalence des plaques qui ne sont pas disjointes forme une sous-variété de classe $C^{r}$ et dimension $k$ de $M$, dite feuille de $\mathcal{L}$.

L'image des ensembles $A \times D^{i} \subset A \times D^{k}$ par les applications $\Phi_{1}=\left.\Phi\right|_{A \times D^{i}}$ définit une lamination de classe $C^{r}$ et dimension $i<k$ dans $M$, dite lamination subordonnée à $\mathcal{L}$. On remarque que la notion de lamination subordonnée est plus faible que la notion de sous lamination.

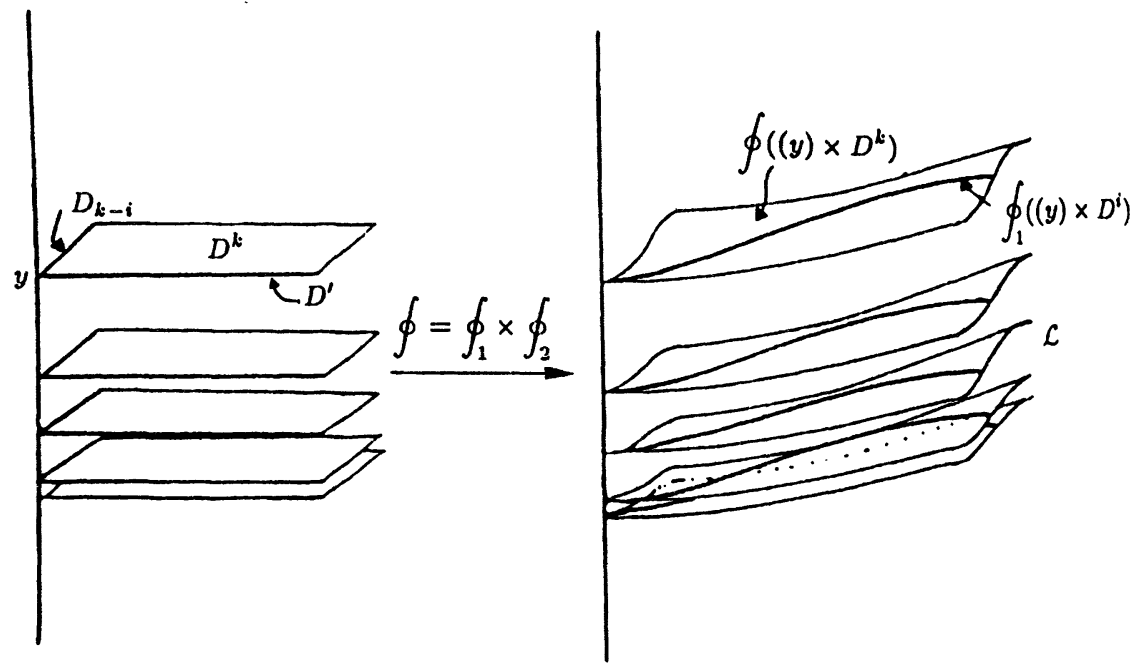

Figure 1

Pour donner un sens à la phrase suivante : "la famille $L_{i}$ de sous-variétés de $M$ de dimension $p_{i}$ converge vers la sous-variété $C$ de dimension $k<p_{i}{ }^{\prime \prime}$, il nous faut maintenant relever des sous-variétés dans des espaces de jets adaptés. 
D'abord on dit que la famille $L_{i}$ converge vers $C$ dans la topologie $C^{\circ}$ si

$$
\lim _{i \rightarrow \infty} D\left(L_{i}, C\right)=\lim _{i \rightarrow \infty}\left(\sup _{x \in C} d\left(x, L_{i}\right)\right)=0
$$

où $d\left(x, L_{i}\right)=\inf _{y \in L_{i}} d(x, y)$ avec d la distance sur $M$.

On remarque que $D$ n'est pas une distance sur $M$. On remarque aussi qu'en particulier $L_{i}$ converge vers $C$ dans la topologie $C^{\circ}$ s'il existe une projection de $C$ sur $L_{i}$ en une $k$-sous-variété $C_{i}$ telle que la distance de Hausdorff de $C_{i}$ à $C$ tende vers 0 . Les courbes $\left\{U C_{i} U C\right\}$ forment une lamination de dimension $k$ (la dimension de $C$ ) et de classe $C^{\circ}$.

Maintenant, on va travailler avec des fibrés en grassmaniennes. Tout fibré en grassmaniennes sur une variété munie d'une métrique riemannienne est aussi muni d'une métrique riemannienne. Sur chacun de ces fibrés, on va considérer la convergence $C^{\circ}$ définie comme ci-dessus.

Soit $\mathcal{G}_{k, n}=\left(\mathcal{G}_{k, n}, \pi, G_{k, n}\right)$ fibré en grassmaniennes des $k$-plans sur $M$. L'application $\pi$ est la projection canonique et par chaque point $x$ de $M$ passe la fibre.

$$
G_{k, n}(x)=\left\{(x, h) / h \text { est un } k \text {-plan dans } I_{x} M\right\}
$$

Posons :

$$
\begin{aligned}
\widetilde{C} & =\left\{\left(x, T_{x} C\right) / x \in C\right\} \quad, \quad \text { et } \\
\tilde{L}_{i} & =\left\{(x, h) / x \in L_{i} \quad \text { et } h \text { est un } k \text {-plan dans } T_{x} L_{i}\right\},
\end{aligned}
$$

pour chaque $L_{i}$.

La sous-variété $\widetilde{C}$ de $\mathcal{G}_{k, n}$ est un graphe au dessus de $C$, donc est de dimension $k$. Chaque $\widetilde{L}_{i}$ est de dimension $p_{i}+k\left(p_{i}-k\right)$.

On dira que la famille $L_{i}$ converge vers $C$ dans la topologie $C^{1}$ si la famille $\widetilde{L}_{i}$ de $\mathcal{G}_{k, n}$ converge vers $\widetilde{C}$ dans la topologie $C^{\circ}$.

Pour itérer ce procédé, on dira que $L_{i}$ converge vers $C$ dans la topologie $C^{r}$ si $\widetilde{L}_{i}$ converge vers $\widetilde{C}$ dans la topologie $C^{r-1}$.

Donnons un exemple pour illustrer ces définitions. Soit $M$ une variété de dimension 3, soit $C$ une courbe dans $M$ et soit $S$ une surface dans $M$. Le fibré $\mathcal{G}_{1,3}=\left(\mathcal{G}_{1,3}, \pi, G_{1,3}\right)$ est de dimension $3+2=5$, où 2 est la dimension de la fibre : On a $\widetilde{C}$ une courbe et $\widetilde{S}$ est une variété de dimension 3 ( $\widetilde{S}$ est une fibré sur $S$ de fibre le cercle). On remarque que le relèvement $\widetilde{\widetilde{C}}$ de $\widetilde{C}$ 
dans $\mathcal{G}_{1,5}$, fibré en grassmaniennes de 1 -plans sur $\mathcal{G}_{1,3}$, est une courbe. Le relèvement $\widetilde{\widetilde{S}}$ de $\widetilde{S}$ dans $\mathcal{G}_{1,5}$ est une sous-variété de dimension $3+2=5$.

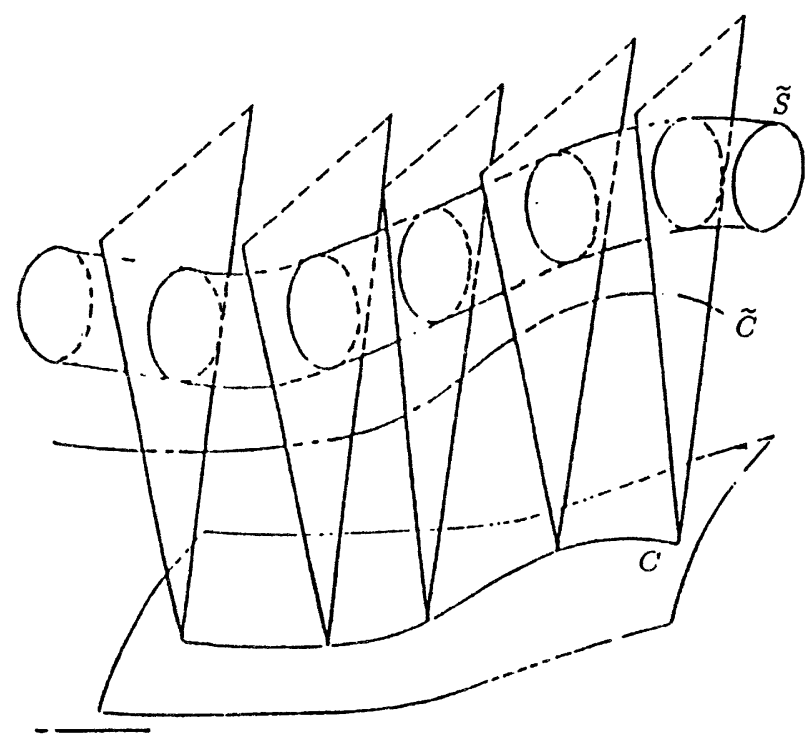

Figure 2

Passons maintenant au rappel de certaines définitions et résultats classiques de la théorie des systèmes dynamiques.

On dit que $f$ satisfait l'Axiome A si l'ensemble non-errant $\Omega(f)$ est hyperbolique et est égal à la fermeture de l'ensemble des points périodiques.

On sait (théorème de la décomposition spectrale) que $\Omega(f)=\Lambda_{1} u \ldots u \Lambda_{k}$ est l'union finie et disjointe d'ensembles fermés transitifs $\Lambda_{i}$, dits pièces basiques. Pour chaque pièce basique $\Lambda$ il existe une décomposition continue en somme directe $\left.T M\right|_{\Lambda}=E^{S} \oplus E^{u}$ tel que pour une métrique adaptée il existe $0<\delta<1$ avec $\left\|\left.d f\right|_{E^{s}}\right\|<\delta$ et $\left\|\left.d f^{-1}\right|_{E^{u}}\right\|<\delta$. Il existe des variétés instables $W^{u}(\Lambda)=\bigcup_{x \in \Lambda} W^{u}(x)$ et stables $W^{S}(\Lambda)=\bigcup_{x \in \Lambda} W^{S}(x)$, où $W^{u}(x)$ est tangente en $x$ à $E^{u}(x)$ et $W^{S}(x)$ est tangente en $x$ à $E^{S}(x)$. On note $\widehat{W}^{u}(\Lambda)=\bigcup_{x \in \Lambda} \widehat{W}^{u}(x)$ avec $\widehat{W}^{u}(x)=W^{u}(x)-x, W_{\varepsilon}^{u}(\Lambda)=\bigcup_{x \in \Lambda} W_{\varepsilon}^{u}(x)$, avec $W_{\varepsilon}^{u}(x)=\left\{y \in W^{u}(x) / d(x, y)<\varepsilon\right\}$, où $d$ est la distance sur la feuille $W^{u}(x)$.

Notons finalement $W_{\text {loc }}^{u}(x)$ un petit voisinage de $x$ dans la variété instable qui passe par $x$. 
Les variétés instables (et stables) sont disjointes et dépendent de façon continue du point dans la topologie $C^{r}$ (théorème de la variété invariante, $[\mathrm{HP}]$ ), et au voisinage d'une pièce basique fournissent une lamination instable (ou stable) de classe $C^{r}$. Cela implique que pour toute pièce basique $\Lambda$ et pour $\delta$ fixé, $W_{\delta}^{u}(\Lambda)$ est une lamination dans un ouvert de $M$. La variété $M$ se décompose en variétés instables (ou en variétés stables) : tout point de $M$ appartient à une variété stable (et aussi a une variété instable) d'une pièce basique: Pour l'étude des variétés invariantes des ensembles hyperboliques voir [HP] ou [S].

Les pièces basiques sont de plusieurs types : attracteurs, répulseurs ou de type selle. Un attracteur $\Lambda$ est un ensemble dont un voisinage $B(\Lambda)$, dit bassin d'attraction, est tel que l'orbite positive de tout point de $B(\Lambda)$ converge vers $\Lambda$. Parmi les types d'attracteurs, les puits sont dits attracteurs triviaux. Un puits est un point périodique dont toutes les valeurs propres associées ont un module plus petit que un. La variété instable associée est de dimension zéro. D'autre part, il existe des attracteurs hyperboliques non triviaux. Les variétés instables des points d'un tel attracteur ont une dimension différente de zéro et sont entièrement contenues dans l'attracteur. Le bassin d'attraction est feuilleté par les variétés stables des points de l'attracteur. Un exemple très connu d'attracteur hyperbolique non trivial est l'attracteur de Plykin ([Pl], [GH]).

Les répulseurs ont un bassin de répulsion de dimension $n$. En particulier il $\mathrm{y} \cdot \mathrm{a}$ des sources, qui ont des points périodiques dont toutes les valeurs propres ont un module plus grand que un.

Les autres pièces basique sont du type selle.

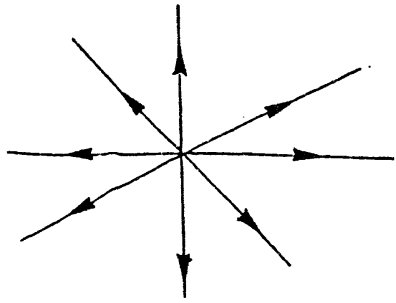

selle

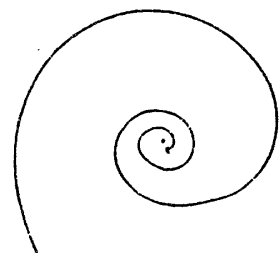

puits

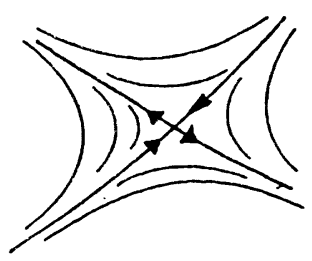

selle 


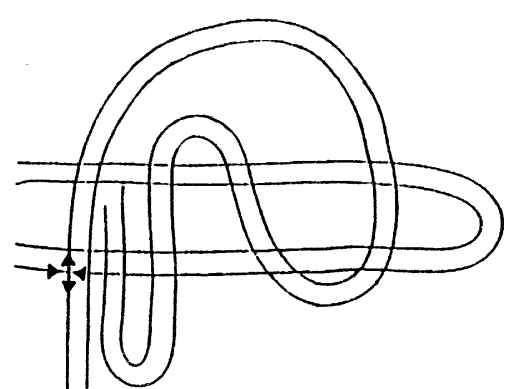

selle

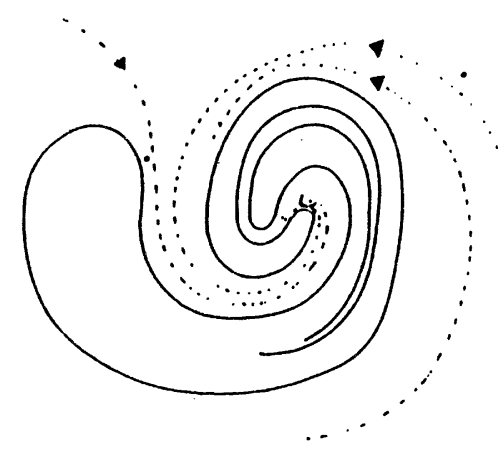

attracteur de Plykin

Figure 3

Pour chaque pièce basique $\Lambda$ il existe un domaine fondamental propre stable, c'est-à-dire un compact $D_{\Lambda}^{S}$ disjoint de $\Lambda$ tel que l'orbite de chaque point de $W^{S}(\Lambda)$ ait au moins un et au plus deux représentants dans $D_{\Lambda}^{S}$ (voir [HPPS]).

Un difféomorphisme $f$ satisfait la condition de transversalité forte si en tout point de la variété $M$ les variétés stables et instables locales sont transverses. On dit que $\Omega(f)$ a un cycle s'il existe $P_{1}, Q_{1} \in \Lambda_{i_{1}}$, $P_{2}, Q_{2} \in \Lambda_{i_{2}}, \ldots, P_{n}, Q_{n} \in \Lambda_{i_{n}}$ tels que $W^{u}\left(P_{j}\right) \cap W^{S}\left(Q_{j+1}\right) \neq \emptyset$ et $W^{u}\left(P_{n}\right) \cap W^{S}\left(Q_{1}\right) \neq \emptyset$ pour tout $j=1, \ldots, n-1$.

Si $f$ satisfait l'Axiome A et la condition de transversalité forte, $\Omega(f)$ n'a pas de cycles et on peut définir un ordre partiel : $\Lambda_{i}>\Lambda_{j}$ si $W^{u}\left(\Lambda_{i}\right) \cap$ $W^{S}\left(\Lambda_{j}\right) \neq \emptyset$. On définit le comportement comp $\left(\Lambda_{i}, \Lambda_{j}\right)=n$ s'il existe une chaîne de pièces basiques $\Lambda_{i}=\Lambda_{i_{1}}>\ldots>\Lambda_{i_{n}}=\Lambda_{j}$ et la longueur de cette chaîne est maximale.

Une référence pour tous ces concepts est le livre de Shub [S].

\section{I - La structure locale}

Pour démontrer le résultat annoncé il suffit de comprendre la structure des variétés invariantes près des pièces basiques non triviales et près des selles. Pour cela, on aura besoin d'une version forte du " $\lambda$-lemma" ([NP]), qu'on démontre dans l'appendice, et d'un théorème qui dit qu'au voisinage 
d'une pièce basique qui n'est pas un attracteur trivial, les variétés instables ont une structure de lamination (comme définie auparavant à l'aide des fibrés en grassmaniennes).

Dans ce paragraphe on va donner d'abord quelques propriétés des difféomorphismes des variétés compactes sans bord de dimension quelconque qui satisfont l'axiome A et la condition de transversalité forte.

Soit $f$ un difféomorphisme de classe $C^{r}, \Lambda$ une pièce basique pour $f$ avec $k=\operatorname{dim} W^{u}(x), x \in \Lambda, k \neq 0$. Soit $D_{\Lambda}^{S}$ un domaine fondamental stable pour $\Lambda$. On remarque que $\Lambda$ peut être un attracteur non trivial.

"-lemma" Soit $N$ une variété fermée de dimension $k$ et classe $C^{r}$ transversale à $D_{\Lambda}^{S}$ et soit $A=N \cap D_{\Lambda}^{S}$. Etant donné $\varepsilon>0$, il existe un voisinage $V$ de $W_{\varepsilon}^{u}(\Lambda)$ avec la propriété suivante : pour tout $\delta>0$ il existe $\bar{n}$ tel que pour tout $n \geq \bar{n}$ les composantes connexes de $f^{n}(N) \cap V$ qui contiennent des points de $f^{n}(A)$ sont $\delta-C^{r}$ proches de $W_{\varepsilon}^{u}(\Lambda)$.

Remarque. - La démonstration du " $\lambda$-lemma" prouve qu'une lamination $\mathcal{L}$ de dimension $k$ et de classe $C^{r}$ transverse à $D_{\Lambda}^{S}$ se prolonge à une lamination dans un voisinage $V$ de $\Lambda$, donné par

$$
\left\{\bigcup_{n \in N} f^{n}(L) \cup V\right\}_{L \in \mathcal{L}} \cup W^{u}(\Lambda) .
$$

Corollaire . - Si $\Lambda_{i}$ et $\Lambda_{j}$ sont des pièces basiques telles que $\Lambda_{i}>\Lambda_{j}$ et si $T$ est un disque fermé coupant transversalement $W^{S}\left(\Lambda_{i}\right), T$ coupe aussi $W^{S}\left(\Lambda_{j}\right)$.

La démonstration de ces résultats est dans l'appendice. Passons à l'étude de la structure locale.

LEMME 0.- Soient $P$ et $Q$ deux points d'une pièce basique $\Lambda$ et soit $A$ un point d'intersection transversale de $W^{u}(P)$ et $W^{S}(Q)$. Le point $A$ appartient alors $\dot{a} \Lambda$.

Démonstration. - Il existe une orbite dense qui approche $P$ et $Q$. Comme les points périodiques sont denses dans $\Lambda$, pour tout $\varepsilon_{i}$ il existe un point périodique $P_{i}$ dont l'orbite rencontre les boules $B_{\varepsilon i}(P)$ et $B_{\varepsilon i}(Q)$ (où $B_{\varepsilon i}(P)$ est une boule dans $M$ ). On peut construire maintenant deux suites $P_{i, 1} \rightarrow P$ et $P_{i, 2} \rightarrow Q$ où, pour tout $i, P_{i, 1}$ et $P_{i, 2}$ sont des points de l'orbite de $P_{i}$.

Soient $\delta_{1}$ et $\delta_{2}$ tels que $A \in W_{\delta_{1}}^{u}(P)$ et $A \in W_{\delta_{2}}^{S}(Q)$ (rappelons que $W_{\delta_{1}}^{u}(P)=\left\{y \in W^{u}(P) / d(y, P)<\delta_{1}\right\}$ où $d$ est la distance géodésique sur la 
feuille $\left.W^{u}(P)\right)$. Le théorème de la variété invariante implique que $W_{\delta_{1}}^{u}\left(P_{i, 1}\right)$ converge vers $W_{\delta_{1}}^{u}(P)$ et $W_{\delta_{2}}^{S}\left(P_{i, 2}\right)$ converge vers $W_{\delta_{2}}^{s}(Q)$ (convergence dans la topologie $C^{r}$ ). On a donc une suite $x_{i}$ de points homocliniques transverses de $W_{\delta_{1}}^{u}\left(P_{i, 1}\right) \cap W_{\delta_{2}}^{S}\left(P_{i, 2}\right)$. La suite $x_{i}$ tend vers $A$ et le point $A$ appartient donc à $\Lambda$.

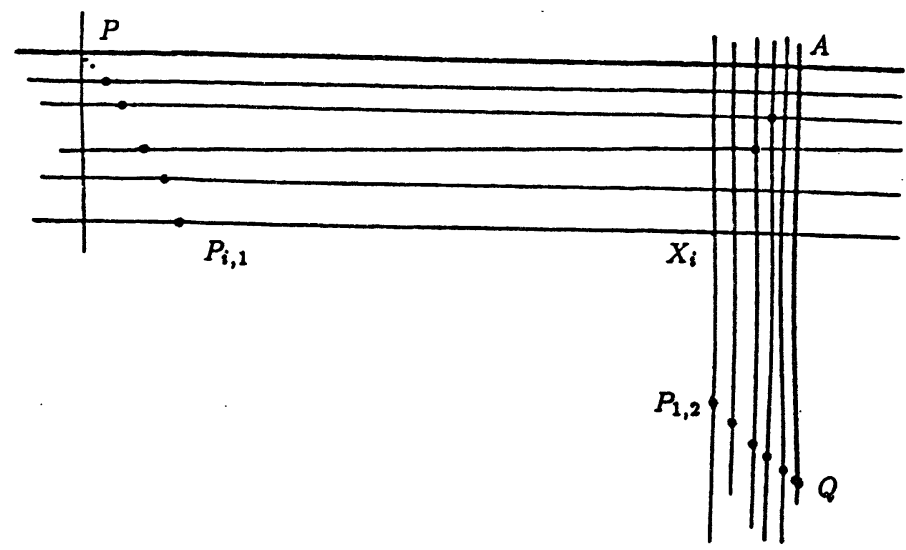

Figure 4

LemMe 1.- (Type Smale, voir [P] page 389).

Pour toute pièce basique $\Lambda$ il existe un voisinage $V$ de $\Lambda$ tel que toute variété instable qui coupe $V$ doit couper $W^{S}(\Lambda)$

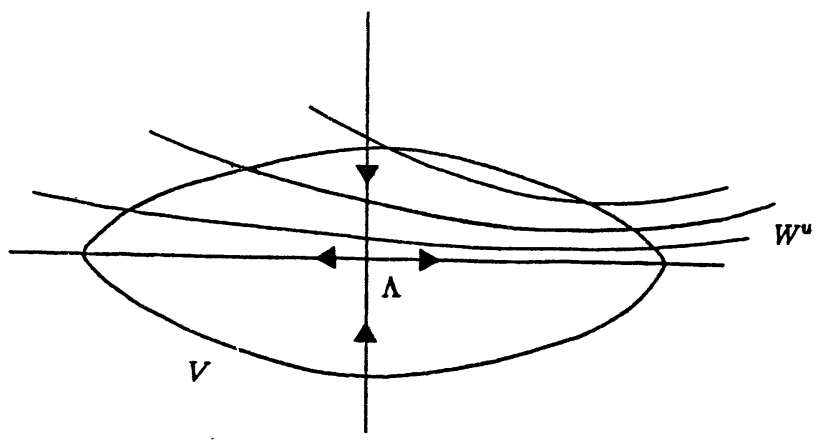

Figure 5 
Démonstration. - Soit $\left\{W_{i}^{u}\right\}$ une famille de variétés instables de points de $\Omega(f)$ et soit $\bar{W}=\overline{\bigcup_{1} W_{i}^{u}}$. Le lemme se déduit des deux affirmations suivantes :

Affirmation 1.1: $\mathrm{Si} \bar{W} \cap W^{u}(\Lambda) \neq \emptyset$, on a :

$\bar{W} \cap \widehat{W}^{S}(\Lambda) \neq \emptyset$ (où $\left.\widehat{W}^{S}(\Lambda)-\Lambda\right)$

Démonstration. - Soit $\nu$ un voisinage quelconque de $\widehat{W}^{S}(\Lambda)$. Alors $A=$ $\bigcup_{n \geq 0} f^{n}(\nu) \cup W^{u}(\Lambda)$ est un voisinage de $W^{u}(\Lambda)$. $n \geq 0$

Comme $W_{i}^{u} \cap W^{u}(\Lambda)=\emptyset$ on a $W_{i}^{u} \cap \bigcup_{n \geq 0} f^{n}(\nu) \neq \emptyset$ et $W_{i}^{u} \cap \nu \neq \emptyset$.

Affirmation 1.2: Si $\bar{W} \cap \widehat{W}^{S}(\Lambda) \neq \emptyset$; il existe $i$ tel que $W_{i}^{u} \cap W^{S}(\Lambda) \neq \emptyset$.

Démonstration. - Soit $z \in \widehat{W}^{S}(\Lambda) \cap \bar{W}$

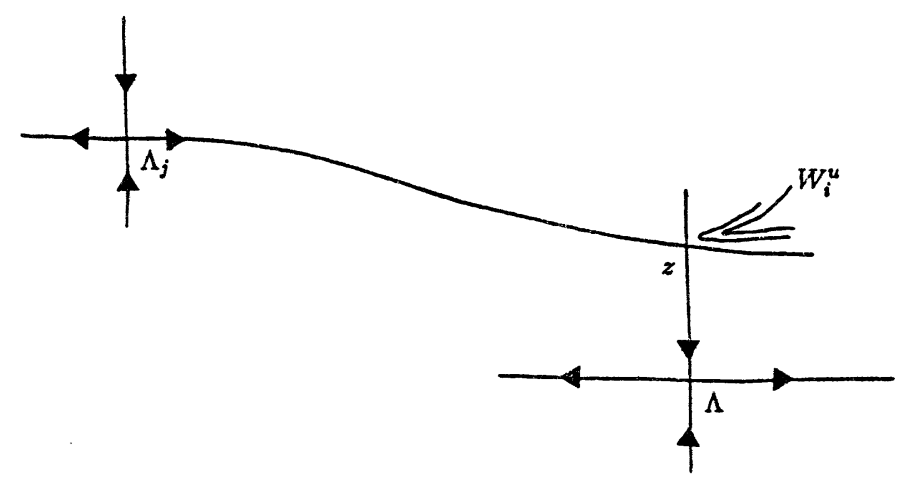

Figure 6

Par la décomposition de $M$ en variétés instables il existe $\Lambda_{j}$ tel que $z$ appartient à $W^{u}\left(\Lambda_{j}\right)$. Si $z$ appartient à $\bigcup_{i} W_{i}^{u}$, c'est fini. Si $\Lambda_{j}=\Lambda, z$ appartient à $\Lambda$, ce qui contredit l'appartenance de $z$ à $\widehat{W}^{S}(\Lambda)$. Si $\Lambda_{j}$ est différent de $\Lambda$ (voir figure 6), z appartient à $W^{u}\left(\Lambda_{j}\right) \cap \bar{W}$ et donc par l'affirmation antérieure $\widehat{W}^{S}\left(\Lambda_{j}\right) \cap \bar{W} \neq \emptyset$.

On répète le raisonnement. Comme on a un nombre fini de pièces basiques, on arrive à une suite $\Lambda_{\ell_{1}}>\Lambda_{\ell_{2}}>\ldots>\Lambda_{j}>\Lambda$ telle que 
$\bigcup_{1} W_{i}^{u} \cap W^{S}\left(\Lambda_{\ell_{1}}\right) \neq \emptyset$. Par le corollaire du " $\lambda$-lemma" on conclut que $\bigcup_{1}^{1} W_{i}^{u} \cap W^{S}(\Lambda) \neq \emptyset$.

THÉORÈme 1.- (Structure locale de lamination).

Pour chaque pièce basique $\Lambda$ qui n'est pas attracteur trivial, étant donné $\varepsilon$ il existe un voisinage de $W_{\varepsilon}^{u}(\Lambda)$ tel que dans ce voisinage les variétés instables varient de façon continue dans la topologie $C^{r}$.

La clé de la démonstration du théorème est la proposition 1 qui a été énoncée dans un article de Newhouse et Palis ([NP], §2). On remarque que la même propriété dans le cas des difféomorphismes de Morse-Smale a permis de démontrer leur stabilité structurelle ([P]).

Soit $\Lambda$ une pièce basique.

Proposition 1.- Etant donné $\varepsilon$, il existe un voisinage de $W_{c}^{u}(\Lambda)$ où les variétés instables qui coupent $W^{S}(\Lambda)$ varient de façon continue dans la topologie $C^{r}$.

Démonstration. - Pour montrer la proposition on va montrer une série de lemmes.

Soit $D_{\Lambda}^{S}$ un domaine fondamental associé à la pièce basique $\Lambda$.

Lemme 2. - Si $\operatorname{comp}\left(\Lambda_{i}, \Lambda\right)=1$, l'ensemble $K=W^{u}\left(\Lambda_{i}\right) \cap D_{\Lambda}^{S}$ est compact.

Démonstration. - Soit $x_{n} \in K, x_{n} \rightarrow x$. Comme $D_{\Lambda}^{S}$ est compact il suffit de montrer que $x \in W^{u}\left(\Lambda_{i}\right)$. Comme $M=\bigcup_{k} W^{u}\left(\Lambda_{k}\right)$ il existe $\Lambda_{r}$ tel que $x \in W^{u}\left(\Lambda_{r}\right)$. Si $\Lambda_{r}=\Lambda_{i}$ est fini. Supposons $\Lambda_{r}$ différent de $\Lambda_{i}$. On a donc un point $x$ dans l'intersection de $W^{u}\left(\Lambda_{r}\right)$ et de $W^{s}(\Lambda)$, i.e., $\Lambda_{r}>\Lambda$.

De plus $W^{u}\left(\Lambda_{r}\right) \cap \overline{W^{u}\left(\Lambda_{i}\right)} \neq \emptyset$. Donc par le lemme $1, W^{u}\left(\Lambda_{i}\right) \cap W^{S}\left(\Lambda_{r}\right) \neq$ $\emptyset$, et donc $\Lambda_{i}>\Lambda_{r}>\Lambda$, ce qui contredit $\operatorname{comp}\left(\Lambda_{i}, \Lambda\right)=1$.

LEMME 3. - Soit $\widetilde{\Lambda}$ une pièce basique. Si $K$ est un compact dans $W^{u}(\tilde{\Lambda})$, l'ensemble $\tilde{K}=\bigcup_{x \in K} W_{\text {loc }}^{u}(x)$ varie de façon continue dans la topologie $C^{r}$.

Démonstration. - Il suffit de montrer qu'il existe $\delta$ tel que $W_{\delta}^{u}(\tilde{\Lambda})$ contient $K$. 
Soit $y_{m} \in K$. Il existe $Q \in \Lambda$ et $\delta_{m}>0$ tels que $y_{m} \in W_{\delta_{m}}^{u}(Q)$. On va choisir un voisinage $B_{\xi}(Q)$ tel que $B_{\xi}(Q)$ soit contenu dans un voisinage $V$ de $\tilde{\Lambda}$ donné par le lemme $1:$ toute variété instable dans $B_{\xi}(Q) \subset V$ doit couper $W^{S}(\widetilde{\Lambda})$, et doit donc couper $W^{S}(Q)$. Il existe $k \in \mathbf{R}$ et $\varepsilon>0$ tels que $f^{-k}\left(B_{\varepsilon}\left(y_{m}\right)\right)$ soit contenue dans $B_{\xi}(Q)$, et que pour tout point $x$ appartenant à $K \cap B_{\varepsilon}\left(y_{m}\right), W_{\text {loc }}^{u}\left(f^{-k}(x)\right)$ coupe $W^{S}(Q)$.

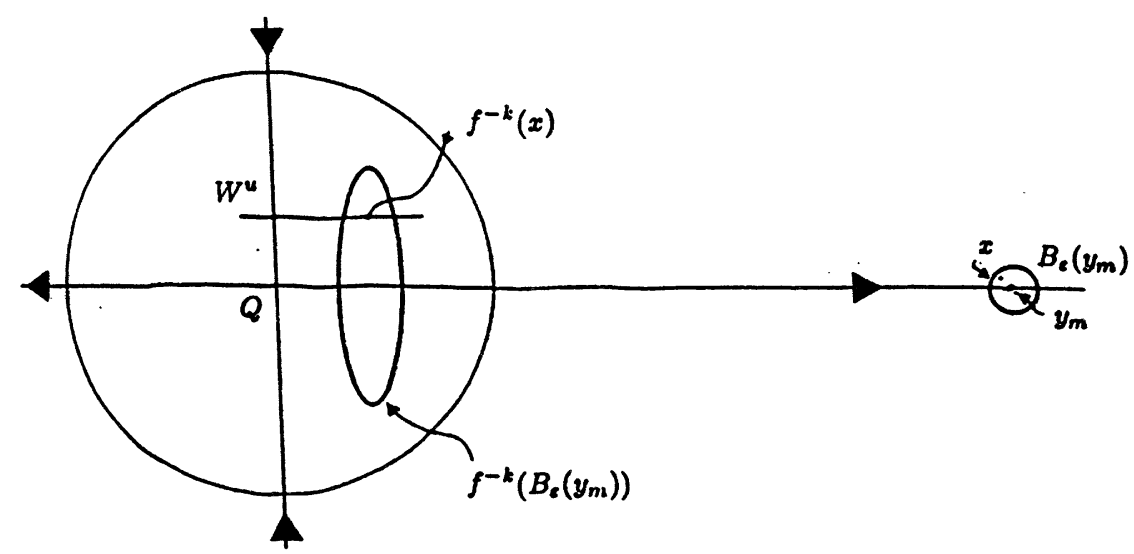

Figure 7

Par le lemme 0 l'ensemble des points $a_{i}=W^{S}(Q) \cap W_{\mathrm{loc}}^{u}\left(f^{-k}\left(x_{i}\right)\right)$, pour tout $x_{i}$ appartenant à $B_{\varepsilon}\left(y_{m}\right) \cap K$, est contenu dans $\Lambda$, et il existe $\delta_{m}$ tel que $K \cap B_{e}\left(y_{m}\right)$ est contenu dans $W_{\delta_{m}}^{u}(\Lambda)$.

Maintenant $\bigcup_{y_{m} \in K} B_{\varepsilon_{m}}\left(y_{m}\right)$ est un recouvrement ouvert de $K$, donc il existe $I=\left(i_{1}, \ldots, i_{\ell}\right)$ tel que $\bigcup_{i \in I} B_{\varepsilon_{i}}\left(y_{i}\right)$ est un recouvrement fini de $K$. Pour $\delta=\max \left\{\delta_{i}\right\}_{i \in I}, W_{\delta}^{u}(\Lambda)$ est une lamination $C^{r}$ qui contient $K$.

Corollaire du lemme 3. - Si comp $\left(\Lambda_{i}, \Lambda\right)=1$, étant donné $\varepsilon$, il existe un voisinage $V$ de $W_{\varepsilon}^{u}(\Lambda)$ où les variétés $\left\{W^{u}\left(\Lambda_{i}\right) \cap V\right\} \cup W_{\varepsilon}^{u}(\Lambda)$ varient 


\section{Ana Cascon}

de façon continue dans la topologie $C^{r}$

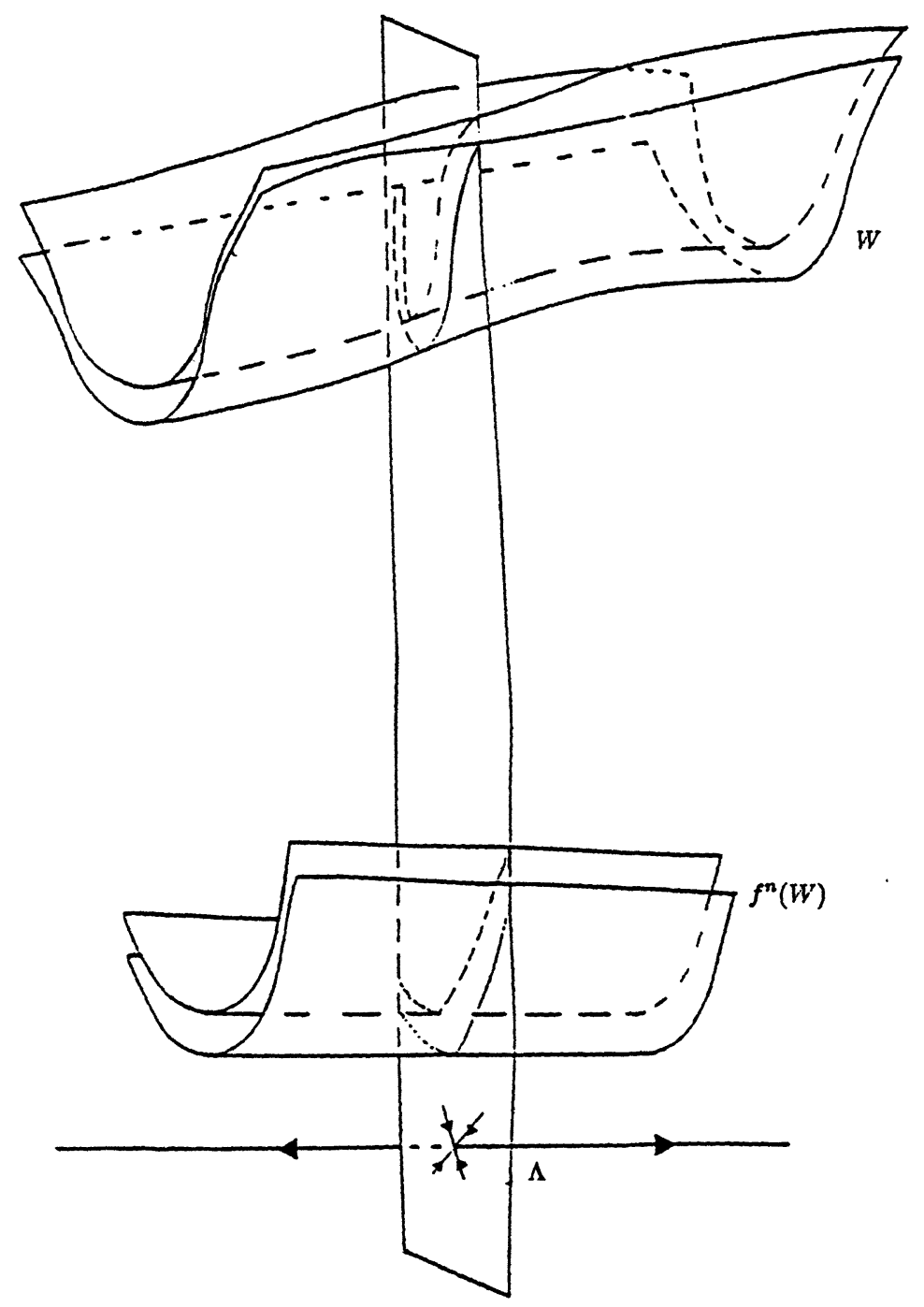

Figure 8 
Démonstration. - Par le lemme 1 il existe un voisinage $V$ de $W_{\varepsilon}^{u}(\Lambda)$ où les variétés de $W^{u}\left(\Lambda_{i}\right)$ coupent $W^{S}(\Lambda)$, il existe un petit voisinage $V^{S}$ d'un domaine fondamental $D_{\Lambda}^{S}$ où les variétés de $W^{u}\left(\Lambda_{i}\right)$ doivent couper $W^{S}(\Lambda)$. De plus $\bigcup_{n \geq 0} f^{n}\left(V^{s}\right) \cup W^{u}(\Lambda)$ est un voisinage de $\Lambda$ où les variétés de $W^{\boldsymbol{u}}\left(\Lambda_{i}\right)$ doivent couper $W^{S}(\Lambda)$. Par le lemme 3 , dans $V^{S}$, les variétés de $W^{u}\left(\Lambda_{i}\right)$ fournissent une lamination $\mathcal{L}$ de classe $C^{r}$ et la dimension de $W^{u}(\Lambda)$. On peut prendre une lamination $\widetilde{\mathcal{L}}$ subordonnée à $\mathcal{L}$, de classe $C^{r}$, de dimension $k$ telle que $\widetilde{\mathcal{L}}$ est transverse à $W^{s}(\Lambda)$. Finalement, par la remarque qui suit

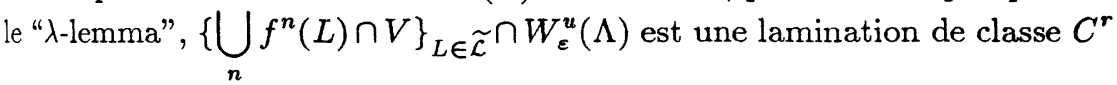
et dimension $k$ dans le voisinage $V$ de $\Lambda$.

On remarque que si $\Lambda_{i}$ et $\Lambda_{j}$ sont deux pièces basiques ayant un comportement 1 par rapport à $\Lambda$ alors $K_{i}=W^{u}\left(\Lambda_{i}\right) \cap D_{\Lambda}^{S}$ et $K_{j}=$ $W^{u}\left(\Lambda_{j}\right) \cap D_{\Lambda}^{s}$ sont disjoints et le corollaire du lemme 3 est vrai pour l'ensemble de pièces basiques qui ont un comportement 1 par rapport à $\Lambda$.

LEMME 4. - Soit $\widetilde{\Lambda}=\bigcup_{\Lambda_{\ell}>\Lambda} \Lambda_{\ell}$. L'intersection de $W^{u}(\widetilde{\Lambda})$ et $D_{\Lambda}^{S}$ varie de fa̧̧on continue dans la topologie $C^{r}$.

Démonstration. - Soit $A=W^{u}(\widetilde{\Lambda}) \cap D_{\Lambda}^{S}$. Le lemme est vrai pour deux pièces basiques de comportement 1 (lemme 3 ). On va faire la démonstration par induction sur la hiérarchie des pièces basiques.

Soit $\Lambda_{i_{1}}, \ldots, \Lambda_{i_{p}}$ les pièces basiques qui ont un comportement 1 par rapport à $\Lambda$. Les ensembles $K_{j}=W^{u}\left(\Lambda_{i j}\right) \cap D_{\Lambda}^{S}$ sont des compacts disjoints. De plus il existe $\delta_{1}, \ldots, \delta_{p}$ tels que $K_{j} \subset W_{\delta j}^{u}\left(\Lambda_{i j}\right), \forall j=1, \ldots, p$.

On va montrer que le lemme est vrai pour les variétés invariantes des pièces basiques de comportement 2. Les étapes suivantes de la récurrence sont analogues.

Supposons que $\Lambda r$ soit une pièce basique avec $\operatorname{comp}(\Lambda r, \Lambda)=2$. Prenons $T_{1}, \ldots, T_{p}$ des petits voisinages tubulaires ouverts de $W_{\delta_{1}}^{u}\left(\Lambda_{i_{1}}, \ldots, W_{\delta_{p}}^{u}\left(\Lambda_{i_{p}}\right)\right.$ tels que $T_{1}, \ldots, T_{p}$ sont disjoints. Soit $T=\bigcup_{j=1, \ldots, p} T_{j}$. Par le corollaire du lemme 3, comme comp $\left(\Lambda_{r}, \Lambda_{i j}\right)$ est au plus un, pour $j$ variant de 1 à $p$, les variétés de $\left(W^{u}\left(\Lambda_{r}\right) \cap T\right) \bigcup_{j=1, \ldots, p} W_{\delta_{j}}^{u}\left(\Lambda_{i j}\right)$ varient de façon continue dans la topologie $C_{r}$.

Il reste à montrer que les variétés de $W^{u}\left(\Lambda_{r}\right)$ qui coupent $D_{\Lambda}^{S}$ en dehors de 
$T$ varient de façon continue dans la topologie $C^{r}$. Pour ça il suffit de montrer que l'ensemble $B=W^{u}\left(\Lambda_{r}\right) \cap\left(D_{\Lambda}^{S}-T\right)$ est compact (alors il existe $\xi$ tel que $W_{\xi}^{u}\left(\Lambda_{r}\right) \supset B$, où $W_{\xi}^{u}\left(\Lambda_{r}\right)$ varie de façon continue dans la topologie $\left.C^{r}\right)$. Soit $x_{n} \in B, x_{n} \rightarrow x$. Comme $D_{j}^{S}-T$ est fermé il faut voir que $x \in W^{u}\left(\Lambda_{r}\right)$. Par la décomposition de $M$ il existe $\Lambda_{\ell}$ tel que $x \in W^{u}\left(\Lambda_{\ell}\right)$. Supposons $\Lambda_{r}$ différent de $\Lambda_{\ell}$, et donc $\overline{W^{u}\left(\Lambda_{r}\right)} \cap W^{u}\left(\Lambda_{\ell}\right)$ est non vide. Le lemme 1 implique donc que $\Lambda_{r}>\Lambda_{\ell}>\Lambda$. Mais comme $\operatorname{comp}\left(\Lambda_{r}, \Lambda\right)=2$ on a $\operatorname{comp}\left(\Lambda_{\ell}, \Lambda\right)=1$ et $x \in T$, ce qui est une contradiction.

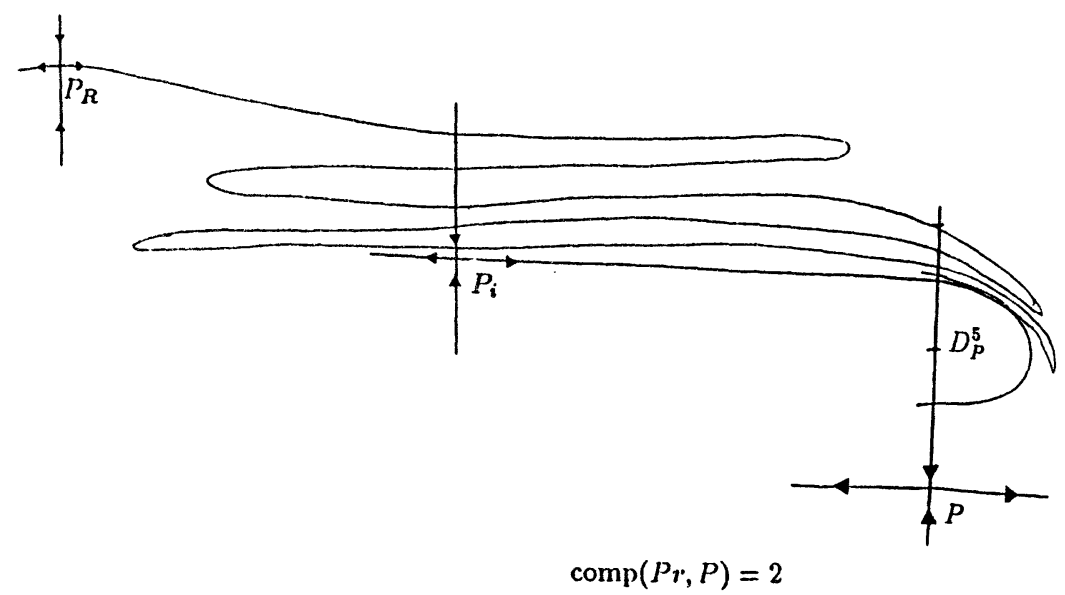

Figure 9

On remarque que maintenant la proposition 1 est un corollaire du lemme 4. Sa démonstration est analogue à la démonstration du corollaire du lemme 3.

Démonstration du Théorème 1. - Par le lemme 1 il existe un voisinage $V$ de $\Lambda$ où toute variété instable coupe $W^{s}(\Lambda)$. Pour les variétés qui coupent $W^{S}(\Lambda)$ le théorème est vrai : c'est la proposition 1.

\section{II - Structure globale}

Soit maintenant $M$ une surface compacte sans bord munie d'un difféomorphisme $f$ de classe $C^{r}, r \geq 2$ qui satisfait l'axiome A et la condition de transversalité forte. 
Géométrie des Variétés Invariantes d'un Difféomorphisme Axiome A et Transversalité

THÉORÈME 2. - Si $x$ est un point d'accumulation de courbure instable alors $x$ est un puits.

Démonstration. - Soit $\left\{W_{n}^{u}\right\}$ une réunion de courbes invariantes instables et soit une suite $x_{n} \in W_{n}^{u}$ avec $x_{n} \rightarrow x$ et $K\left(x_{n}\right) \rightarrow \infty$ quand $n \rightarrow \infty$, où $K\left(x_{n}\right)$ est la courbure géodésique de $W_{n}^{u}$ en $x_{n}$.

Par la décomposition de $M$ il existe un point $P$ d'une pièce basique $\Lambda$ tel que $x \in W^{u}(P)$. On a trois possibilités : soit $\Lambda$ est un répulseur, soit $\Lambda$ est un attracteur, soit $\Lambda$ de type selle.

Si $\Lambda$ était répulseur, dont la variété instable globale est un ouvert de dimension deux, alors il existerait un $n$ tel que $W^{u}(P) \cap W_{n}^{u} \neq \emptyset$, ce qui est absurde car des variétés instables ne se coupent pas.

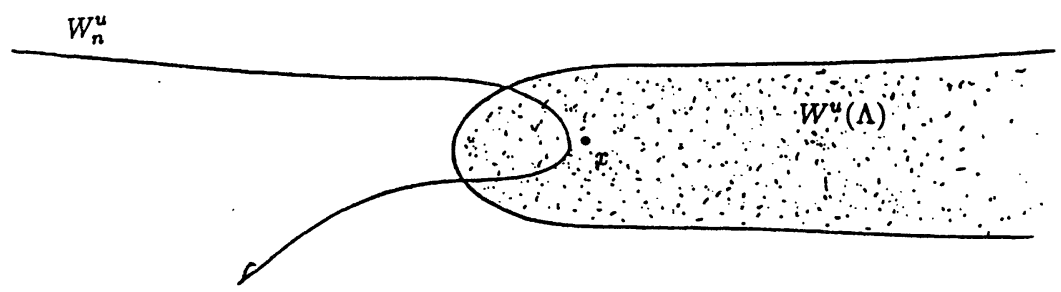

Figure 10

Voyons que $\Lambda$ ne peut pas être une pièce basique du type selle ni un attracteur non trivial : un point d'accumulation de courbure $x \in W^{u}(\Lambda)$ est tel qu'il existe $\varepsilon>0$ avec $x \in W_{\varepsilon}^{u}(\Lambda)$. Mais ça contredit la structure locale de lamination donné par le théorème 1 . Alors $\Lambda$ est un puits et $\Lambda=x$ est le point d'accumulation de courbure.

Donnons quelques exemples de difféomorphismes qui satisfont l'axiome $A$ et la condition de transversalité forte.

Exemple 1. - Les difféomorphismes de Morse-Smale sont un type particulier : leur ensemble non errant est fini, et donc égal à l'ensemble des points périodiques. Dans [C] et [CL] on rencontre plusieurs exemples de difféomorphismes de Morse-Smale qui possèdent des points d'accumulation de courbure. Les deux situations type qu'on rencontre aux voisinages des puits sont illustrées par les figures 11 et 12 . Dans les deux cas $P_{1}$ et $P_{2}$ sont des puits, $P$ et $Q$ des selles. 

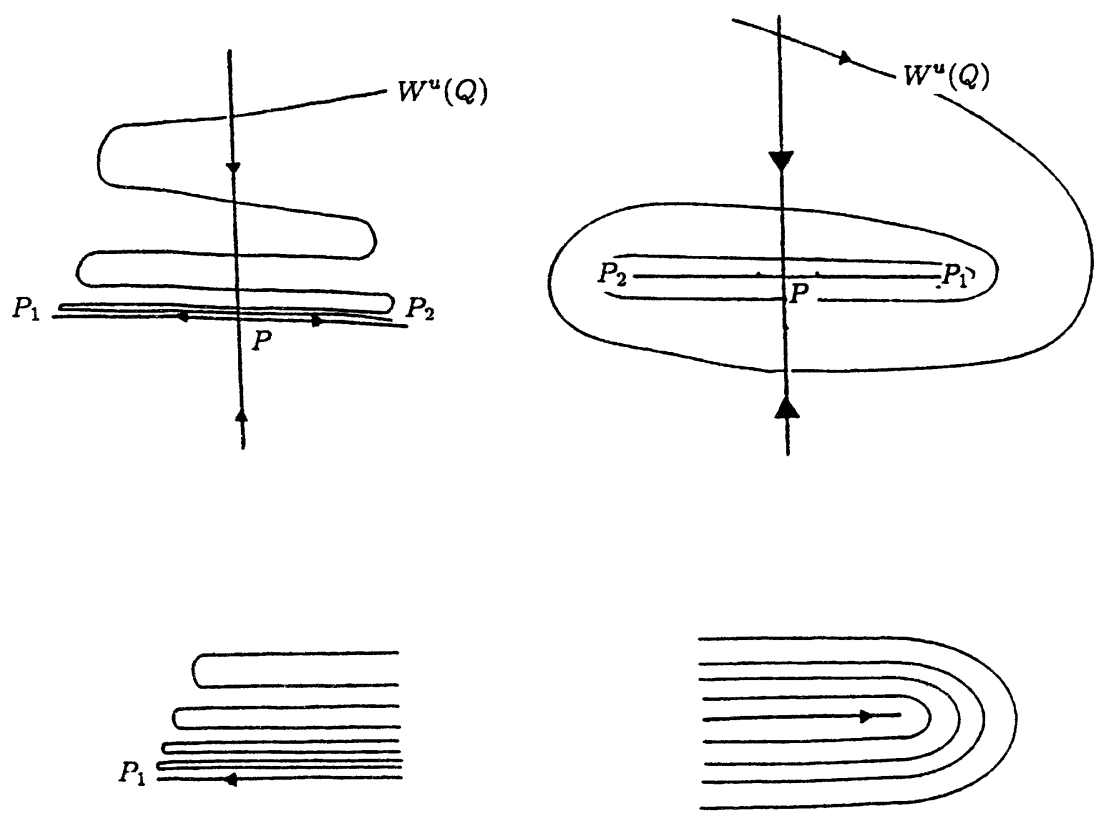

$P_{1}$ est un point d'accumulation $P_{1}$ est un point d'accumulation de courbure du type accordéon Figure 11 de courbure du type épine Figure 12

Ces exemples peuvent être obtenus à partir de difféomorphismes définis par le temps 1 d'un champ de vecteurs modifiés par une isotopie locale (comme dans la figure 12) ou par un twist de Dehn (comme dans la figure 13). À partir d'une suite de telles opérations on peut avoir toutes les combinaisons possibles de ces situations ([L]).

Exemple 2. - Le fer à cheval de Smale (voir $[\mathrm{S}]$ ou $[\mathrm{GH}]$ ).

Soit $f$ un difféormorphisme de $S^{2}$ qui transforme un carré $Q$ dans un fer à cheval comme dans la figure 13 . 


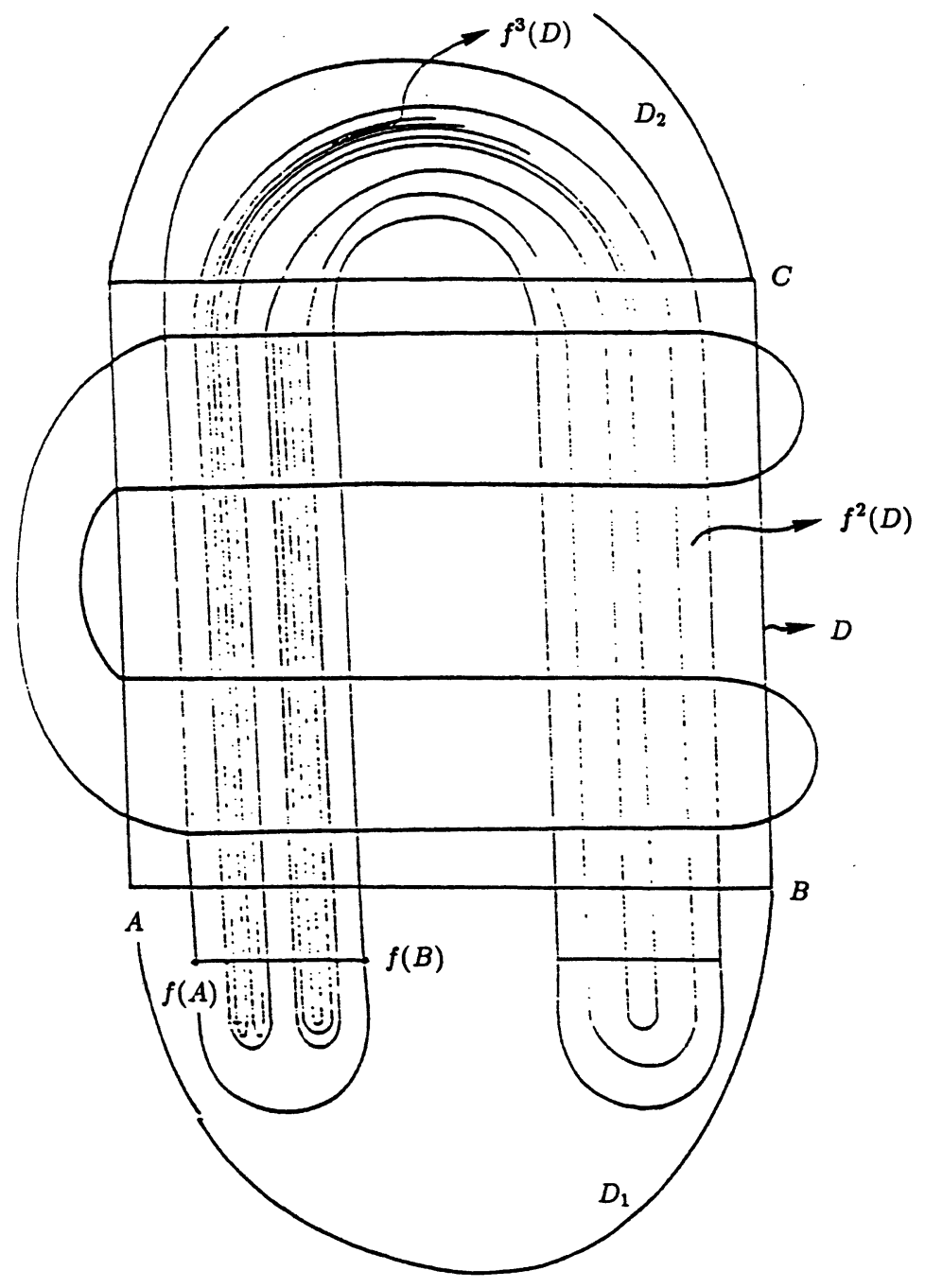

Figure 13

L'ensemble $\Lambda=\bigcap_{n \in Z} f(Q)$ est un ensemble de Cantor hyperbolique. Les variétés instables (ou stables) de $\Lambda$ sont de la forme : produit d'un ensemble 


\section{Ana Cascon}

de Cantor par un intervalle et sur $D_{2}$ fournissent une lamination en semicercles parallèle au bord de $f(Q) \cap D_{2}$. Dans le disque $D_{1}$, les variétés instables définissent une suite infinie de boucles de plus en plus étroites et emboitées. Le point d'accumulation de courbure est bien un puits.

On remarque que l'existence d'un point homoclinique transversal (càd, point d'intersection transverse des variétés stable et instable d'un même point périodique) donne une structure du type du fer à cheval de Smale.

\section{III - Des exemples dans le bord de l'ensemble "Axiome A et Transversalité Forte" qui ne satisfont pas le théorème 2}

Exemple 1. - Sur la variété instable d'un point $Q$ appartenant à une pièce basique non triviale associé à un point de selle dissipatif on fait une modification pour créer une orbite de tangence homoclinique entre $W^{u}(Q)$ et $W^{S}(Q)$.

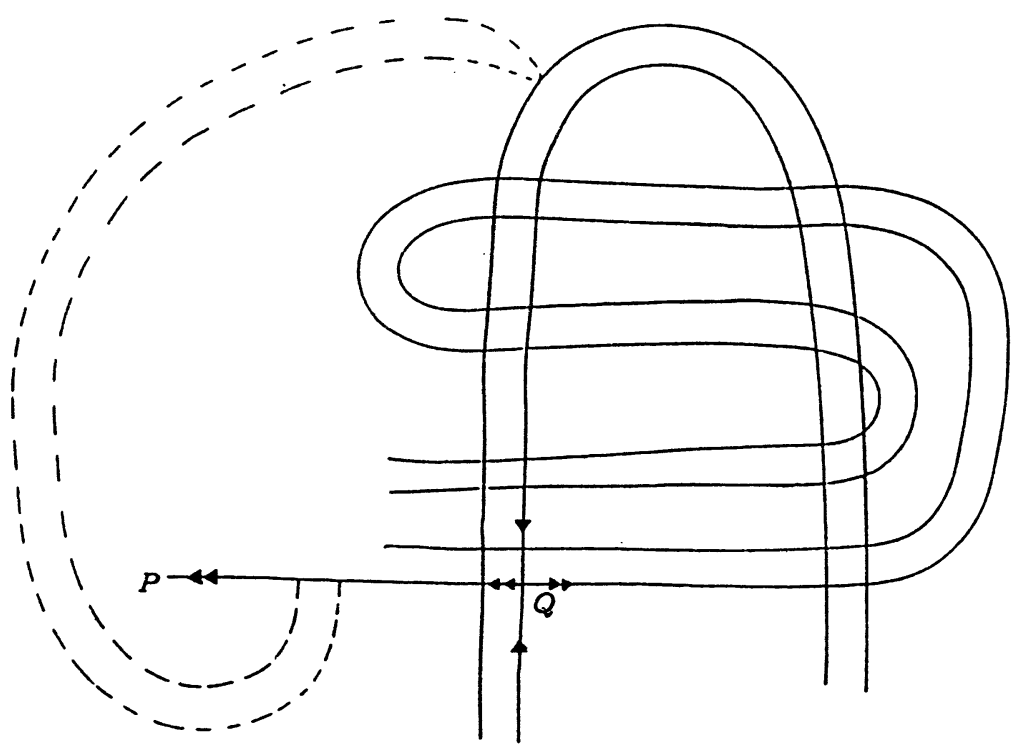

Situation initiale avec en pointillé le chemin suivi par la modification Figure 14 


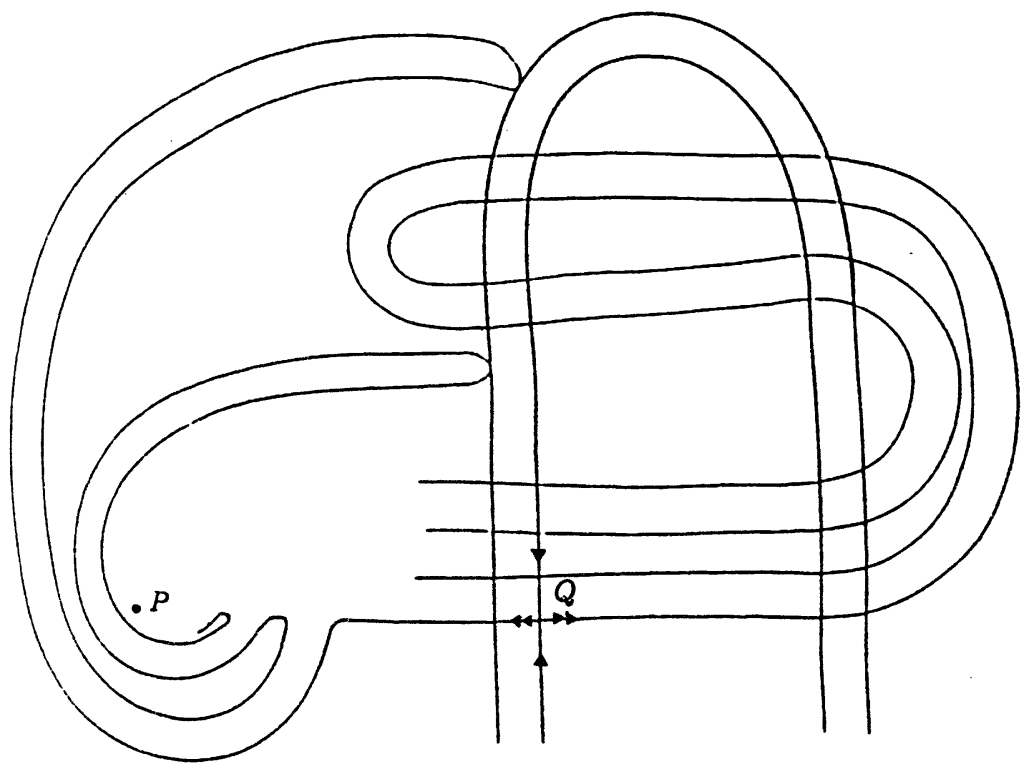

Situation finale

Figure 15

Cette bifurcation a été étudié par Palis et Takens dans [PT] où il a été démontré que cette orbite de tangence homoclinique était la seule nonerrance crée le long de la bifurcation. On voit que la selle $Q$ est maintenant point d'accumulation de courbure.

Exemple 2.- Un difféomorphisme avec un point hétéroclinique de tangence.

Un difféomorphisme avec un nombre fini de points périodiques hyperboliques, dont l'ensemble non-errant est fini et dont les variétés stables et instables de points périodiques distincts se coupent transversalement à l'exception d'une orbite de tangence est dans le bord de l'ensemble de difféormorphismes de Morse-Smale.

On va voir un exemple où il y a une courbe invariante qui s'accumule sur un point hétéroclinique de tangence. On va démontrer, suivant une remarque de W. de MELo, que la courbure de cette courbe invariante s'accumule soit sur toute une composante connexe d'une autre courbe invariante, soit au moins sur un sous-ensemble infini et discret de cette courbe, suivant que le rapport de certaines valeurs propres soit irrationnel ou pas. 


\section{Ana Cascon}

Pour cela, on reprend les idées de J. PALIs $\left[\mathrm{P}_{2}\right]$ et W. de Melo [M] utilisées pour trouver le module de stabilité lié à un point hétéroclinique de tangence. Dans notre exemple, on n'utilise aussi qu'un voisinage des variétés considérées.

\section{Construction de l'exemple}

Soit une surface munie d'un difféomorphisme $f$ qui possède un point hétéroclinique de tangence entre $W^{u}(P)$ et $W^{S}(Q)$ tel que la variété instable d'une selle $R$ s'accumule vers $z$ (on a donc $\operatorname{com}(R, Q)=2$ ) (voir figure 16)

Soient $\lambda$ et $\mu$ les valeurs propres de $d f_{p}, \bar{\lambda}$ et $\bar{\mu}$ les valeurs propres de $d f_{Q}$ et supposons $0<\lambda<1,0<\bar{\lambda}<1, \bar{\mu}$ et $\mu>1$.

On peut prendre $z$ dans un voisinage $V(Q)$. de $Q C^{1}$-linéarisable (pour l'existence d'un tel voisinage voir $[\mathrm{H}]$ ou $[\mathrm{B}]$ ). Supposons que l'image de $z$ par l'application qui linéarise soit un contact du type quadratique (par exemple, c'est le cas quand $z$ est un contact du type quadratique et qu'il existe une linéarisation $C^{2}$, ce qui est une condition générique). Dans un voisinage $V(P)$ de $P C^{1}$-linéarisable prenons une transversale $L$ à $W^{u}(P)$ en un point de l'orbite de $z$, disons en $f^{-S}(z)$, et prenons un voisinage $S$ de $w$ dans $W^{u}(R)$, où $w$ apparartient à $W^{u}(R) \cap W^{S}(P)$. On peut prendre $L$ et $S$ tels que leur intersection soit non vide et tels que $f^{S}(L)$ soit dans le voisinage $V(Q)$.

Dans les coordonnées locales $x, y$ de $V(P)$, on écrit $w=(x, 0)$. En prenant tous les itérés positifs de $S$ on induit une suite $\left\{\left(\bar{x}_{n}, \bar{y}_{n}\right)\right\}$ sur $L$ telle que $\bar{x}_{n}=\lambda^{n} x_{n}, \bar{y}_{n}=\mu^{n} y_{n}$ où $\left\{\left(x_{n}, y_{n}\right)\right\}$ est une suite sur $S$ avec $x_{n}$ qui tend vers $X$ et $y_{n}$ qui tend vers zéro (voir figure 17 ).

On itère $L$ par $f^{S}$ et on obtient une suite $\left\{f^{S}\left(\bar{x}_{n}, \bar{y}_{n}\right)\right\}$ sur $f^{S}(L)$ qui tend vers $z$ (voir figure 16). Dans les coordonnées locales $b, a$ de $V(Q)$ on peut écrire $f^{S}\left(\bar{x}_{n}, \bar{y}_{n}\right)=\left(b_{n}, a_{n}\right)$. De plus $b_{n}=E_{n} \lambda^{n} x_{n}$ avec $E_{n}$ qui tend vers $E$, où $E$ exprime la dérivée de $f^{S}$ dans la direction verticale.

Proposition .- Il existe une suite $\left\{\left(\bar{b}_{n}, \bar{a}_{n}\right)\right\}$ dans $W^{u}(R)$ qui tend vers $z$ telle que si $f^{m}\left(\bar{b}_{n}, \bar{a}_{n}\right) \in V(Q)$ alors

$$
\lim _{\substack{n \rightarrow \infty \\ m \rightarrow \infty}} K\left(f^{m}\left(\bar{b}_{n}, \bar{a}_{n}\right)\right)=\infty
$$

où $K$ est la courbure géodésique.

Corollaire . - S'il existe des sous-suites $m_{j}$ et $n_{j}$ telles que $f^{m_{j}}\left(\bar{b}_{n_{j}}, \bar{a}_{n_{j}}\right)$ converge vers un point de $W^{u}(Q)$, ce point sera un point d'accumulation de courbure de $W^{u}(R)$. 
Géométrie des Variétés Invariantes d'un Difféomorphisme Axiome A et Transversalité

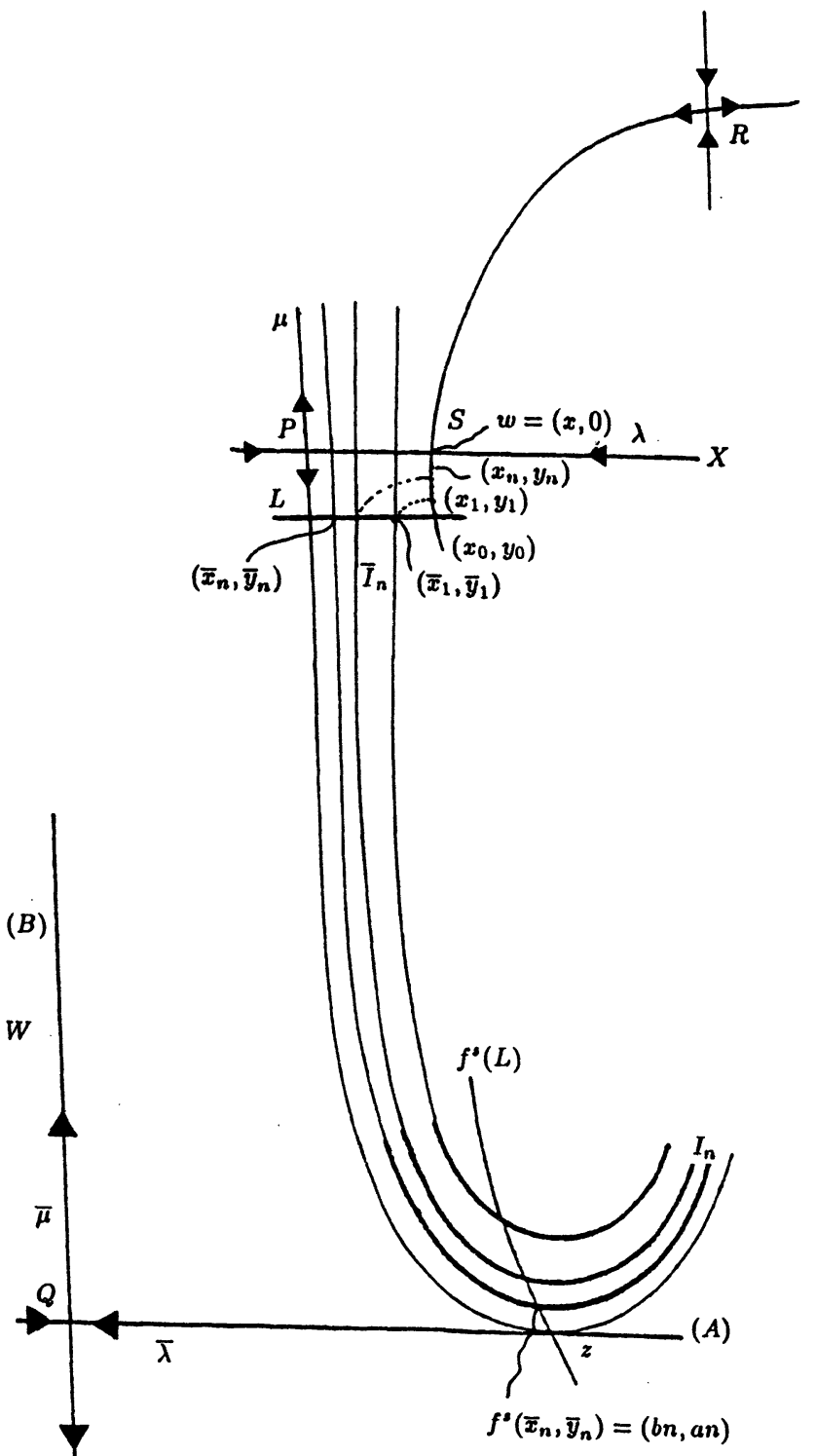

Figure 16

Démonstration de la proposition

Prenons des voisinages $\bar{I}_{n}$ de $\left(\bar{x}_{n}, \bar{y}_{n}\right)$ dans $W^{u}(R)$ tels que leurs images 


\section{Ana Cascon}

$I_{n}=f^{S}\left(\bar{I}_{n}\right)$ dans $V(Q)$ soient comme dans la figure 18.

Soit $h$ l'application $C^{1}$ qui linéarise le difféomorphisme $f \operatorname{sur} V(Q)$, c'està-dire

$$
h: V(Q) \rightarrow B \times A \in \mathbf{R}^{2}
$$

$$
h f h^{-1}=L: \mathbf{R}^{2} \rightarrow \mathbf{R}^{2} \text { est une application linéaire de matrice }\left(\begin{array}{cc}
\bar{\mu} & 0 \\
0 & \bar{\lambda}
\end{array}\right)
$$

On peut prendre $h$ tel que les directions de $B$ et de $A$ soient perpendiculaires.

On va travailler sur $B \times A \subset \subset \mathbf{R}^{2}$ et on va trouver des arcs dont la variation de l'angle tangent est bornée entre $\pi / 2-\delta$ et $\pi / 2+\delta$, avec $\delta$ petit, et dont la longueur d'arc tend vers zéro. Ces propriétés sont invariantes par une conjugaison de classe $C$ et donc sur $V(Q)$ on trouve une suite d'arcs qui possèdent des points dont la courbure tend vers l'infini.

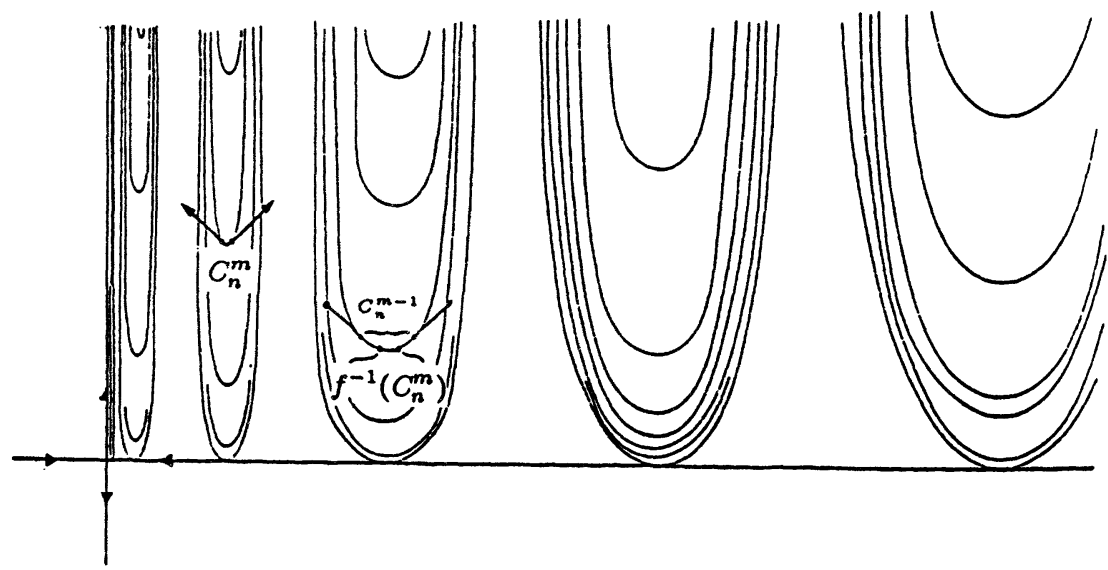

Figure 17

Soit donc $I_{n}=h\left(I_{n}\right)$ et puisque le contact $h(z)$ est quadratique on peut écrire

$$
I_{n}(t)=\left(t, \alpha_{n} t^{2}+t^{2} \varepsilon(t)\right)
$$

avec 
Géométrie des Variétés Invariantes d'un Difféomorphisme Axiome A et Transversalité

$$
\lim _{t \rightarrow 0} \varepsilon(t)=0, \varepsilon \text { de classe } C^{2} \text { et } \lim _{n \rightarrow \infty} \alpha_{n}=\alpha \neq 0 .
$$

et

$$
I_{n}^{m}(t)=L^{m}\left(I_{n}(t)\right)=\left(t, \frac{\bar{u}^{m}}{\bar{\lambda}^{2 m}}\left[\alpha_{n} t^{2}+t^{2} \varepsilon\left(\frac{t}{\bar{\lambda}^{m}}\right)\right]\right)
$$

Sur chaque $I_{n}^{m}(t)$ on prend un arc $C_{n}^{m}$ tel que les extrémités de $C_{n}^{m}$ soient les points $I_{n}^{m}\left(\frac{\bar{\lambda}^{2 m}}{2 \alpha_{n} \bar{\mu}^{m}}\right)$ et $I_{n}^{m}\left(-\frac{\bar{\lambda}^{2 m}}{2_{\alpha_{n} \bar{\mu}^{m}}}\right) \quad$ (voir figure 17)

A chaque pas il faut prendre $m$ de sorte que $C_{n}^{m} \subset B \times A$, mais on remarque que si $n$ croît, on trouve des valeurs de $m$ de plus en plus grandes qui vérifient cette condition.

Par un calcul, on voit que la variation de l'angle entre la tangente à $C_{\boldsymbol{n}}^{\boldsymbol{m}}$ et l'horizontale est bornée entre $\pi / 2-\delta$ et $\pi / 2+\delta$, avec $\delta$ petit.

La longueur de l'arc $C_{n}^{m}$ est proche de $\frac{\bar{\lambda}^{2 m}}{2 \bar{\mu}^{m} \alpha_{n}}\left(\sqrt{2}+\frac{1}{2} \log \left(\frac{\sqrt{2}+1}{\sqrt{2}-1}\right)\right)$ qui tend vers zéro quand $m$ tend vers l'infini.

Donc les arcs $h^{-1}\left(C_{n}^{m}\right)$ ont une variation de l'angle entre la tangente et la direction de $W^{S}(Q)$ bornée près d'une valeur $\theta$, et la longueur de ces arcs tend vers zéro quand $m$ et $n$ crôissent. Donc il existe au moins un point $\left(\bar{b}_{n}^{m}, \bar{a}_{n}^{m}\right)$ sur $h^{-1}\left(C_{n}^{m}\right)$ tel que la courbure $K\left(\bar{b}_{n}^{m}, \bar{a}_{n}^{m}\right)$ est grande et tend vers l'infini avec $m$ et $n$.

Maintenant il reste à voir que $\lim _{n \rightarrow \infty} f^{m}\left(\bar{b}_{n}^{m}, \bar{a}_{n}^{m}\right)=z$.

Pour chaque $n, L^{-m}\left(C_{n}^{m}\right)$ est un arc sur $I_{n}(t)$ dont la longueur est de l'ordre de $\frac{1}{\bar{\mu}^{m} \alpha n}+\frac{\bar{\lambda}^{2 m}}{\bar{\mu} \alpha_{n}}$ qui tend vers zéro quand $m$ tend vers l'infini.

De plus les points tels que la tangente à $V_{n}(t)$ soit parallèle à l'axe horizontal sont contenus dans les $\operatorname{arcs} L^{-m}\left(C_{n}^{m}\right)$.

Donc les $\operatorname{arcs} h^{-1}\left(L^{-m}\left(C_{n}^{m}\right)\right) \subset V_{n}$ ont aussi une longueur qui tend vers zéro quand $m$ tend vers l'infini et contiennent les points où la tangente à $V_{n}$ est parallèle à la direction de $W^{S}(Q)$, et $\lim _{\substack{m \rightarrow \infty \\ n \rightarrow \infty}} h^{-1}\left(L^{-m}\left(C_{n}^{m}\right)\right)=z$.

$$
\begin{gathered}
\text { Comme }\left(\bar{b}_{n}, \bar{a}_{n}\right)=f^{-m}\left(\bar{b}_{n}^{m}, \bar{a}_{n}^{m}\right) \subset h^{-1}\left(L^{-m}\left(C_{n}^{m}\right)\right), \text { alors } \\
\qquad \lim _{n \rightarrow \infty}\left(\bar{b}_{n}, \bar{a}_{n}\right)=z .
\end{gathered}
$$


Pour trouver les points de $W^{u}(Q)$ qui sont des points d'accumulation de courbure de $W^{u}(R)$, il faut trouver les sous-suites $m_{j}$ et $n_{j}$ qui tendent vers l'infini et telles que $f^{m_{j}}\left(\bar{b}_{n_{j}}, \bar{a}_{n_{j}}\right)$ convergent vers un point de $W^{u}(Q)$. Comme la deuxième coordonnée tend forcément vers zéro on ne va travailler qu'avec la première coordonnée.

On a deux suites $\left(b_{n}, a_{n}\right)$ et $\left(\bar{b}_{n}, \bar{a}_{n}\right)$ sur $V_{n}$ telles que

$$
\begin{aligned}
& \lim _{n \rightarrow \infty}\left(b_{n}, a_{n}\right)=z \\
& \lim _{n \rightarrow \infty}\left(\bar{b}_{n}, \bar{a}_{n}\right)=z
\end{aligned}
$$

On peut donc écrire $\bar{b}_{n}=c_{n} b_{n}$ avec $\lim _{n \rightarrow \infty} c_{n}=c$ et on a donc l'équation

$$
\bar{b}_{n}=c_{n} E_{n} \cdot \lambda^{n} x_{n}
$$

De plus $f^{m}\left(\bar{b}_{n}, \bar{a}_{n}\right)=\left(\bar{b}_{n}^{m}, \bar{a}_{n}^{m}\right)$ avec $\bar{b}_{n}^{m}=\bar{\mu}^{m} \bar{b}_{n}$

Finalement $\quad \bar{b}_{n}^{m}=\bar{\mu}^{m} c_{n} E_{n} \lambda^{n} x_{n}$

et

$$
\log \bar{b}_{n}^{m}=m \log \bar{\mu}+n \log \lambda+\log c_{n}+\log E_{n}+\log x_{n}
$$

où

$$
\begin{aligned}
& \lim _{n \rightarrow \infty} \log c_{n}=\log c \text { avec } c \neq 0 \\
& \lim _{n \rightarrow \infty} \log E_{n}=\log E \text { avec } E \neq 0 \\
& \lim _{n \rightarrow \infty} \log x_{n}=\log X \text { avec } X \neq 0
\end{aligned}
$$

On voit qu'une condition nécessaire pour que $\log \vec{b}_{n}^{m}$ converge (et donc pour que $\bar{b}_{n}^{m}$ converge loin de zéro) est que

$$
\lim \frac{m}{n}=\frac{-\log \lambda}{\log \bar{\mu}}
$$

TIIÉORÈme A.- Si $\frac{-\log \lambda}{\log \bar{\mu}}$ est irrationnel alors tous les points d'une composante connexe de $W^{u}(Q)-Q$ sont des points d'accumulation de courbure de $W^{u}(R)$.

Démonstration.-Prenons $\frac{P_{n}}{q_{n}}$ la suite des meilleurs approximations de $\frac{-\log \lambda}{\log \mu}$ par des rationnels, qui vérifie 
On en déduit l'inégalité

$$
\left|\frac{p_{n}}{q_{n}}+\frac{\log \lambda}{\log \mu}\right|<\frac{c}{q_{n}^{2}}
$$

$$
\left|p_{n} \log \bar{\mu}+q_{n} \log \lambda\right|<\frac{c \log \bar{\mu}}{q_{n}}
$$

Les suites $p_{n}$ et $q_{n}$ tendent vers l'infini avec $n$ et

$$
\lim _{n \rightarrow \infty} p_{n} \log \bar{\mu}+q_{n} \log \lambda=0
$$

Pour les suites $s=\left(p_{n}+A\right)$ et $t=\left(q_{n}+B\right)$ avec $A$ et $B$ des entiers quelconques on a

$$
\lim _{n \rightarrow \infty} \log \bar{b}_{t}^{S}=A \log \bar{\mu}+B \log \lambda+\log E+\log X
$$

qui est un ensemble dense dans $\mathbf{R}$ quand $A$ et $B$ parcourent les entiers.

En particulier pour tout point $x$ de $W \cap V(Q)$, on trouve des sous-suite $\bar{s}$ et $\bar{t}$ tels que $f^{\bar{s}}\left(\bar{b}_{\bar{t}}, \bar{a}_{\bar{t}}\right)$ converge vers $x$, où $W$ est la composante connexe de $W^{u}(Q)-Q$ qui est "du même côté" de $W^{s}(Q)$ que les arcs $I_{n}$ (voir figure 37)

Donc tout point de $W$ est point d'accumulation de courbure de $W^{u}(R)$.

THÉORÈME B. - Si $-\frac{\log \lambda}{\log \mu}$ est rationnel, alors l'ensemble de points de $W^{u}(Q)$ qui sont des points d'accumulation de courbure de $W^{u}(R)$ est au moins infini et discret.

Démonstration. - Comme $-\log \lambda$ est positif et $\log \bar{\mu}$ est positif, on a $\frac{-\log \lambda}{\log \bar{\mu}}=\frac{p}{q}$ avec $p$ et $q$ des entiers positifs et premiers entre eux. Donc il existe des entiers $\alpha$ et $\beta$ positifs tels que $\beta q-\alpha p=1$ et pour tout $z \in \mathbf{Z}$ on a l'égalité

$$
[z \cdot p+\beta] q-[z \cdot q+\alpha] p=1
$$

et pour tout $z$ :

$$
\{c[z \cdot p+\beta] q-c[z \cdot q+\alpha] p\}=c \quad, \quad \forall c \in \mathbf{N}
$$

Donc pour les sous-suites $c[z p+\beta]=s$ et $c[z q+\alpha]=t$, qui tendent vers l'infini quand $z$ tend vers l'infini,

$$
\lim _{\substack{s \rightarrow \infty \\ t \rightarrow \infty}} \log \bar{b}_{t}^{s}=c \frac{\log \mu}{q}+\log X+\log E
$$


Lorsque $c$ décrit $\mathbf{N}$, on a un ensemble sur $W^{u}(Q)$ de points d'accumulation de courbure de $W^{u}(R)$ discret et infini.

\section{APPENDICE - LE “ $\lambda$-LEMMA"}

La première version du " $\lambda$-lemma" a été démontrée par J. PALIS ([P]). Il a montré que les itérés par un difféomorphisme d'une transversale à la variété stable d'un point hyperbolique s'approchent dans la topologie $C^{1}$ de la variété instable du même point. Dans $[\mathrm{C}]$ on a démontré, à partir des suggestions de R. LANGEvin et F. TAKENS, le " $\lambda$-lemma" pour la topologie $C^{2}$. Pour le faire on a relevé le difféomorphïsme dans le fibré unitaire tangent. Cette méthode ne pouvait pas être généralisée pour les variétés de dimension plus grande que deux.

La version démontrée ci-dessus a été énoncée par S. NEwhouse et J. PALIS ([NP]), page 56). La démonstration donnée ici m'a été suggérée par J. PaLIS. L'idée principale est de relever le difféomorphisme dans un fibré en grassmaniennes bien choisi.

Soit donc $M$ une variété de dimension $n$ munie d'un difféomorphisme $f$ de classe $C^{r}, r \geq 1$. Soit $\Lambda$ une pièce basique pour $f$ avec $k=\operatorname{dim} W^{u}(x)$, $x \varepsilon \Lambda, k \neq 0$. Soit $D^{s}$ un domaine stable pour $\Lambda$. On remarque que $\Lambda$ peut être un attracteur non trivial.

" $\lambda$-lemma" : soit $N$ une variété fermée de dimension $k$ et classe $C^{r}$ transversale à $D^{s}$. Soit $A=N \cap D^{s}$. Etant donné $\varepsilon>0$ il existe un voisinage $V$ de $W_{\varepsilon}^{u}(\Lambda)$ avec la propriété suivant : pour tout $\delta>0$ il existe $\bar{n}$ tel que pour tout $n \geq \bar{n}$ les composantes connexes de $f^{n}(N) \cap V$ qui contiennent des points de $f^{n}(A)$ sont $\delta-C^{r}$ proches de $W_{\varepsilon}^{u}(\Lambda)$.

Remarque. - Il découle de la transitivité de $\Lambda$ que $W_{\varepsilon}^{u}(\Lambda)$ est contenue dans la fermeture de $\bigcup_{n} f^{n}(N) \cap V$.

Corollaire . - Si $\Lambda_{i}$ et $\Lambda_{j}$ sont des pièces basiques telles que $\Lambda_{i}>\Lambda_{j}$ et si $T$ est une transversale $\grave{a} W^{s}\left(\Lambda_{j}\right)$ alors $T$ coupe aussi $W^{s}\left(\Lambda_{j}\right)$.

Démonstration du corollaire. - Soit $x$ tel que $W^{u}\left(\Lambda^{i}\right)$ et $W^{s}\left(\Lambda_{j}\right)$ sont transverses en $x$. Soit tel que $x \varepsilon W_{\delta}^{u}\left(\Lambda_{i}\right)$. Alors pour $n$ assez grand, $f^{n}(T)$ est $C^{r}$ proche de $W_{\delta}^{u}\left(\Lambda_{i}\right)$, donc coupe $W^{s}\left(\Lambda_{i}\right)$.

Démonstration $d u$ " $\lambda$-lemma". - On va faire une induction sur la classe de différentiabilité. 


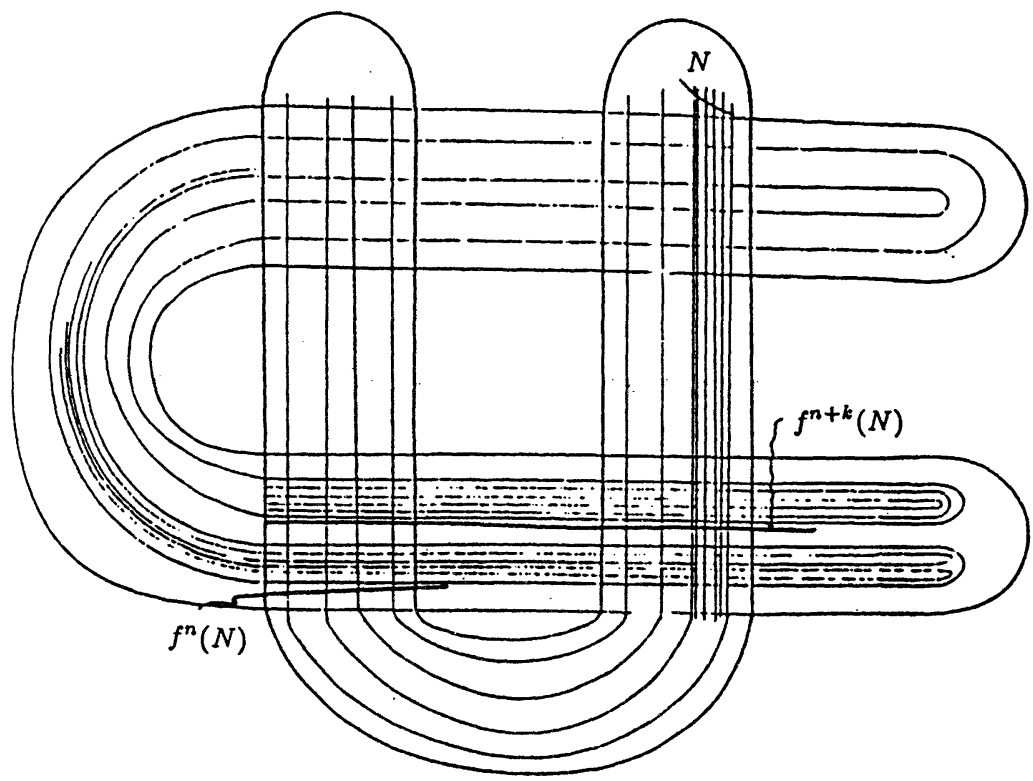

Figure 18

Pour montrer la proximité $C^{\circ}$ on remarque que pour tout voisinage $\nu$ de $\Lambda$ on a :

$$
\bigcap_{n \geq 0} f^{n}(\nu)=W^{u}(\Lambda)
$$

Donc si $N$ est une transversale à $W^{s}(\Lambda)$ dans $\nu, f^{n}(N)$ converge dans la topologie $C^{\circ}$ vers $W^{u}(\Lambda)$.

Montrons maintenant la proximité $C^{i}$. Pour cela, on va relever le difféomorphisme dans un fibré en grassmaniennes au dessus d'un voisinage $V$ de $\Lambda$. Chaque point du fibré sera un couple $(x, h)$ avec $x$ point de $V$ et $h$ un $k$-plan dans $T_{x} V$. Le difféomorphisme $f$ se relèvera en un difféormorphisme $F=(f, \widehat{d f})$ de ce fibré et $F$ aura une pièce basique $\widetilde{\Lambda}$ qui se projette sur la pièce basique $\Lambda$. La transversale $N$ se relèvera en une transversale $\tilde{N}$ à $W^{s}(\widetilde{\Lambda})$. Les itérés par $F$ de $\widetilde{N}$ vont donc s'approcher de $W^{u}(\widetilde{\Lambda})$ dans la topoligie $C^{\circ}$.

En d'autres termes, les itérés des points et les $k$-plans tangents à $N$ seront 


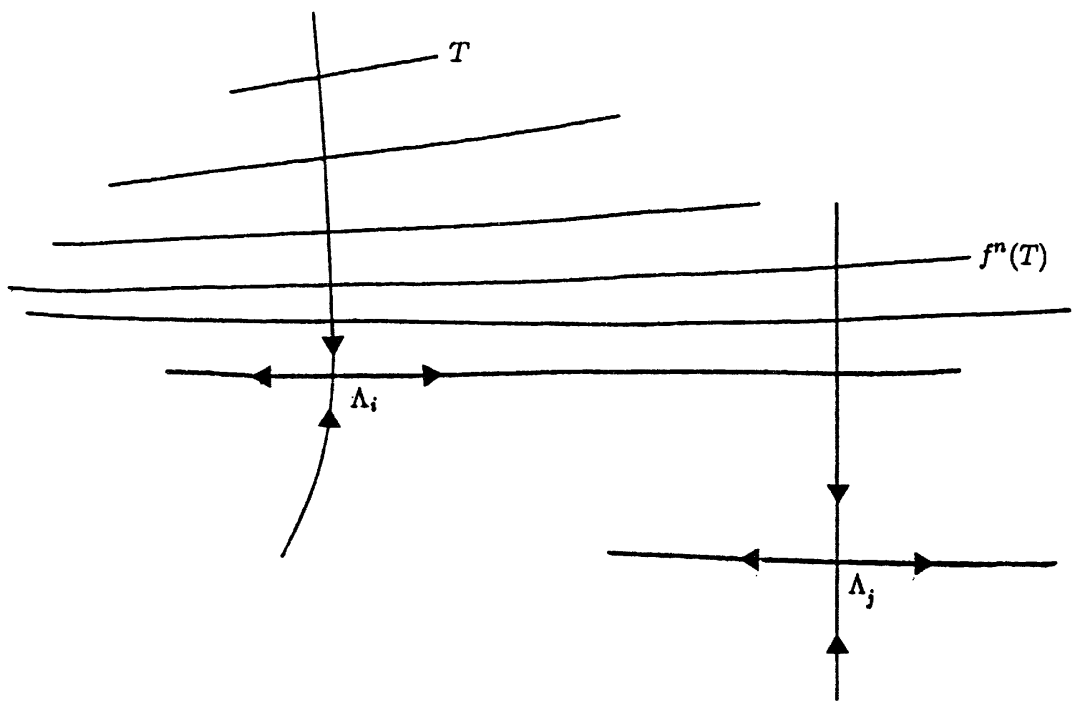

Figure 19

$C^{\circ}$ proches des points et $k$-plans tangents à $W^{u}(\Lambda)$.

Passons à notre construction. Sur la pièce basique $\Lambda$ il existe une décomposition du fibré tangent en somme directe de sous fibrés stable $E^{g}$ et instable $E^{u}$ tels que pour tout point $x$ de $\Lambda$ :

$$
E^{s}(x)=T_{x} W^{s}(\Lambda) \quad \text { et } \quad E^{u}(x)=T_{x} W^{u}(\Lambda)
$$

On peut prolonger cette décomposition de façon continue à des fibrés $E^{s}$ et $E^{u}$ f-invariants dans un voisinage $V$ de $\Lambda$ ([HPPS]).

Sur $V$ on a le fibré $\left(\mathcal{G}_{k}, \pi, G_{k}\right)=\mathcal{G}_{k}$ en grassmaniennes de $k$-plans dont $\pi$ est la projection canonique et dont la fibre passant par un point $x$ de $V$ est :

$$
G_{k}(x)=\left\{(x, h) / h \in T_{x} V \quad \text { et } \quad \operatorname{dim} h=k\right\}
$$

Soit l'application de classe $C^{r-1}$

$$
\begin{aligned}
F: & \mathcal{G}_{k} \rightarrow \mathcal{G}_{k} \\
& (x, h) \rightarrow\left(f(x) ; \widehat{d f}_{x}(h)\right) ;
\end{aligned}
$$


Géométrie des Variétés Invariantes d'un Difféomorphisme Axiome A et Transversalité

où $\hat{d f}_{x}: G_{k}(x) \rightarrow G_{k}(f(x))$ est l'application induite par :

$d f_{x}: T_{x} M \rightarrow T_{f(x)} M$

On pose :

$\tilde{\Lambda}=\left\{\left(x, E^{u}(x)\right) / x \in \Lambda\right\}$

L'ensemble $\widetilde{\Lambda}$ est $F$-invariant et se projette injectivement sur $\Lambda$, donc $\widetilde{\Lambda}$ est une orbite dense. Pour démontrer que $\widetilde{\Lambda}$ est une pièce basique pour une structure hyperbolique de $F$, on va définir des ensembles $\widetilde{W}^{s}$ et $\widetilde{W}^{u}$. On démontrera que ces ensembles sont les variétés invariantes associées à $\widetilde{\Lambda}$.

Tout d'abord on va montrer que $\widetilde{W}^{s}$ et $\widetilde{W}^{u}$ sont des variétés de classe $C^{r-1}, F$-invariantes, transverses et qui se coupent suivant $\widetilde{\Lambda}$. De plus on verra que sur $\widetilde{W}^{u}, \mathrm{~d} F$ est une contraction et que sur $\widetilde{W}^{u}, \mathrm{~d} F$ est une dilatation. On aura donc une décomposition en somme directe du fibré tangent à $\mathcal{G}_{k}$ sur $\widetilde{\Lambda}$ en des sous-fibrés $\widetilde{E}^{s}$ et $\widetilde{E}^{u}$ définis par

$$
\widetilde{E}^{s}(x)=T_{x} \widetilde{W}^{s}, \widetilde{E}^{u}(x)=T_{x} \widetilde{W}^{u} \text {, pour tout } x \in \widetilde{\Lambda} .
$$

Cette décomposition sera continue car $\widetilde{W}^{s}$ et $\widetilde{W}^{u}$ seront de classe $C^{r-1}$.

Définissons donc

$\widetilde{W}^{s}=\left\{(x, h) / x \in W^{s}(\Lambda) \cap V ; h \in T_{x} V\right.$ avec $h$ transverse à $\left.T_{x} W^{s}(\Lambda)\right\}$

$\widetilde{W}^{u}=\left\{\left(x, E^{u}(x) / x \in W^{u}(\Lambda) \cap V\right\}\right.$

L'ensemble $\widetilde{W}^{s}$ est une sous-variété de classe $C^{r-1}$ et dimension $(n-$ $k)+k(n-k)$. Un atlas de $\widetilde{W}^{s}$ est formé par les cartes $\left(V_{i} \times F_{j}, \psi_{i} \times \varphi_{j}\right)$ où $\left(V_{i}, \psi_{i}\right)$ est un atlas $C^{r}$ de $W^{s}(\Lambda)$ tel que $\mathcal{G}_{k \mid V_{i}}$ soit un fibré trivial et où chaque $F_{j}$ est un ouvert dans une fibre passant par un point de $V_{i}$. On a :

$$
\begin{aligned}
\Psi_{i} \times \varphi_{j}: V_{i} \times F_{j} & \rightarrow R^{n-k} \times \mathcal{L}\left(E^{u}(x), E^{s}(x)\right) \\
(x, h) & \rightarrow\left(\Psi_{i}(x), \varphi_{h}\right)
\end{aligned}
$$

où $h$ est le graphe de l'application linéaire

$$
\varphi h: E^{u}(x) \rightarrow E^{s}(x) .
$$

L'ensemble $\widetilde{W}^{u}$ est variété de classe $C^{r-1}$ et de dimension $k:$ un voisinage de $\left(x, E^{u}(x)\right)$ dans $\widetilde{W}^{u}$ est de la forme $\left\{\left(y, E^{u}(y)\right) / y \in A\right\}$ avec $A$ voisinage de $x$ dans $W^{u}(\Lambda)$, et donc est un graphe au dessus de $A$.

Les variétés $\widetilde{W}^{s}$ et $\widetilde{W}^{u}$ sont $\mathrm{F}$-invariantes car $h$ est transverse à $T_{x} W^{s}(\Lambda)$ si et seulement si $d f(h)$ est transverse à $T_{f(x)} W^{s}(\Lambda)$. On note que tout point de l'intersection de $\widetilde{W^{s}}$ et $\widetilde{W}^{u}$ est dans $\widetilde{\Lambda}$. 


\section{Ana Cascon}

Pour voir que $\widetilde{W}^{s}$ et $\widetilde{W}^{u}$ sont transverses on utilise les trivialisations données plus haut.

On vérifie aisément que pour tout point $y$ de $\widetilde{\Lambda}$ :

$$
T_{y} \widetilde{W^{s}} \cap T_{y} \widetilde{W}^{u}=\{0\}
$$

Il reste à vérifier que sur $\widetilde{W^{s}}, d F$ est une contraction et que sur $\widetilde{W}^{u}, d F$ est une dilatation.

Calculons l'application linéaire

$$
\begin{aligned}
& d F: T \mathcal{G}_{k} \rightarrow T \mathcal{G}_{k} \\
& d F_{(x, h)}: T_{(x, h)} \mathcal{G}_{k} \rightarrow T_{(f(x), \widehat{d f}(h)) \mathcal{G}_{k}}
\end{aligned}
$$

On va calculer $d F$ sur la carte de $\widetilde{W}$ obtenue en repérant un point $(x, h)$ de $\widetilde{W}^{s}: h$ est le graphe de l'application linéaire :

$$
\varphi_{h}: E^{u}(x) \rightarrow E^{s}(x)
$$

La première coordonnée de l'application $F$ ne dépend pas du $k$-plan $h$ et quelque soit le choix de trivialisation locale on a :

$$
d F_{(x, h)}=\left(\begin{array}{cc}
d f_{x} & { }^{*} \\
0 & \frac{\partial}{\partial \varphi} \widehat{d f}_{x, \varphi_{k}}
\end{array}\right)
$$

On rappelle que sur $V$ voisinage de $\Lambda$ la décomposition en somme directe donné par $E^{u}$ et $E^{s}$ est $f$-invariante. Dans ces coordonnées l'application $d f$ est donnée par la matrice :

$$
d f_{x}=\left(\begin{array}{cc}
B(x) & 0 \\
0 & A(x)
\end{array}\right)
$$

où $d f_{\left.\right|_{E^{u}}}=B, d f_{\left.\right|_{E^{0}}}=A$ avec $\|B\| \geq \delta^{-1}>1$

$$
\|A\| \leq \delta<1
$$

pour une métrique riemannienne convenablement choisie dans $M$.

L'image de $h$ par $\widehat{d f}_{x}$ est donnée par le graphe de l'application linéaire :

$$
\begin{aligned}
A \varphi_{h}^{B^{-1}}: E^{u}(f(x)) \rightarrow E^{s}(f(x)), \text { car : } & \\
\widehat{d f}_{x}(h)=\widehat{d f}_{x}\left\{\left(y, \varphi_{h}(y)\right) / y \in E^{u}(x)\right\} & =\left\{\left(B_{y}, A \varphi_{h}(y)\right) / y \in E^{u}(x)\right\} \\
& =\left\{\left(y, A \varphi_{h} B^{-1}(y) / y \in E^{u}(f(x))\right\}\right.
\end{aligned}
$$

L'application $\widehat{d f}_{x}$ est linéaire dans cette carte et donc sa dérivée est

$$
\frac{\partial}{\partial \varphi} \widehat{d f}_{x, \varphi_{h}}(v)=A v B^{-1}
$$


Géométrie des Variétés Invariantes d'un Difféomorphisme Axiome A et Transversalité sur $\widetilde{W^{s}}$ on a donc

$$
d F_{(x, h)}(w, v)=\left(\begin{array}{cc}
d f_{x}(w) & 0 \\
0 & A(x) v B^{-1}(x)
\end{array}\right)
$$

L'application $d f$ est contractante sur $W^{s}(\Lambda)$. De plus l'application

$$
v \rightarrow A v B^{-1}
$$

est contractante et $d F_{\left.\right|_{W}}$. est une contraction.

On peut démontrer de façon analogue que $d F_{\widetilde{W}^{u}}$ est une dilatation.

Le difféomorphisme $F$ a donc une pièce basique $\widetilde{\Lambda}$ telle que $W^{s}(\widetilde{\Lambda})=\widetilde{W}^{s}$ et $W^{u}(\widetilde{\Lambda})=\widetilde{W}^{u}$.

Il nous reste à relever la transversale $N$ à $W^{s}(\Lambda)$, en une transversale $\tilde{N}$ $\grave{a} W^{s}(\widetilde{\Lambda})$, et appliquer la proximité $C^{\circ}$ aux itérés par $F$ de $\tilde{N}$.

Soit donc $N$ une variété fermée de dimension $k$ transverse à $W^{s}(\tilde{\Lambda})$.

Posons :

$$
\widetilde{N}=\left\{\left(x, T_{n} N\right) / n \in N\right\}
$$

La variété $\widetilde{N}$ est un graphe au dessus de $N$, donc de dimension $k$. De plus $\tilde{N}$ et $W^{s}(\tilde{\Lambda})$ sont transverses, car ses projections $\pi\left(W^{s}(\widetilde{\Lambda})\right)$ et $\pi(\tilde{N})$ sont transverses.

On applique maintenant le " $\lambda$-lemma" $C^{\circ}$ : quand $n$ tend vers l'infini, $F^{n}(\tilde{N})_{c} a p V(\tilde{\Lambda})$ converge $C^{\circ}$ vers $W_{\varepsilon}^{u}(\tilde{\Lambda})$, où $V(\widetilde{\Lambda})$ est un voisinage de $W_{\varepsilon}^{u}(\tilde{\Lambda})$. Donc $f^{n}(N) \cap V$ converge $C^{1}$ vers $W_{\varepsilon}^{u}(\Lambda)$. On voit immédiatement que le même raisonnement prouve que le " $\lambda$-lemma" $C^{k}$ implique le " $\lambda$ lemma" $C^{k+1}$ pour tout $k<r$.

\section{Références}

[B] BELITSKII (G.R.). - Equivalence and normal forms of germs of smooth mappings, Russian Math. Surweys. $33: 1,1978$, P. 107-177, de Uspekhi Mat. Nauk. $33: 1$, 1978, p. 95-155.

[C] CASCON (A.). - Géométrie des Courbes Invariantes d'un Difféomorphisme, Thèse $3^{\text {ième }}$ cycle, Univ. de Dijon, 1984 .

[CL] CAsCon (A.) and LANGevin (R.). - A Labyrinth and Other Ways to Lose One's Way., Singularities and Dynamical Systems, editor SN Pnevmatikos, North Holland, 1985 . 
[GH] Guchenheimer (J.) and Holmes (P). - Non linear Oscillations, Dynamical Systems and Bifurcations of Vector Fields. Springer.

[H] Hartman (P.). - On local homeomorphisms of Euclidean Spaces, Bol. Soc. Math. Mexicana 25, 196o, p. 220-221.

[HP] Hirsh (M) and Pugh (C.).- Stable Manifolds and Hyperbolic sets, Global Analysis 14 (Proced. Am. Math. Soc., Providence RI 1970).

[HPPS] Hirsh (M), Palis (J.), Pugh (C.) and Shub (M.). - Neighborhoods of Hyperbolic sets, Inventiones Math. 91970.

[L] LANGevin (R.). - Vers une classification des difféomorphismes de Morse-Smale, en préparation.

[M] de MELo (W.).- Moduli of Stability of two-dimensional Diffeormorphisms, Topology 19, 1980, p. 9-21.

[NP] Newhouse (S.) and Palis (J.). - Cycles and Bifurcation Theory, Astérisque 31, 1976.

[P] Palis (J.).- On Morse-Smale Dynamical Systems, Topology 8, 1969.

$\left[\mathrm{P}_{2}\right]$ Palis (J.). - A differentiable Invariant of Topological Conjugary and Moduli of Stability, Astérisque 51, 1978, p. 335-346.

[PL] PLYKIN (R.V.). - Sources and Sinks of A-Diffeomorphisms of Surfaces Math. URSS Sbornik 23, 1974, $\mathrm{n}^{\circ} 2$.

[PT] Palis (J.) and Takens (F.).- Hyperbolicity and the creation of Homoclinic Orbits, Pré-Publication IMPA.

[S] Shub (M.). - Stabilité Globale des Systèmes Dynamiques Astérique 58, 1978.

(Manuscrit reçu le 17 janvier 1989) 